\title{
147.
}

\section{A MEMOIR ON THE SYMMETRIC FUNCTIONS OF THE ROOTS OF AN EQUATION.}

[From the Philosophical Transactions of the Royal Society of London, vol. CxLvII. for the year 1857, pp. 489-499. Received December 18, 1856,-Read January 8, 1857.]

There are contained in a work, which is not, I think, so generally known as it deserves to be, the "Algebra" of Meyer Hirsch [the work referred to is entitled Sammlung von Beispielen Formeln und Aufgaben aus der Buchstabenrechnung und Algebra, 8vo. Berlin, 1804 (8 ed. 1853), English translation by Ross, 8vo. London, 1827] some very useful tables of the symmetric functions up to the tenth degree of the roots of an equation of any order. It seems desirable to join to these a set of tables, giving reciprocally the expressions of the powers and products of the coefficients in terms of the symmetric functions of the roots. The present memoir contains the two sets of tables, viz. the new tables distinguished by the letter $(a)$, and the tables of Meyer Hirsch distinguished by the letter $(b)$; the memoir contains also some remarks as to the mode of calculation of the new tables, and also as to a peculiar symmetry of the numbers in the tables of each set, a symmetry which, so far as I am aware, has not hitherto been observed, and the existence of which appears to constitute an important theorem in the subject. The theorem in question might, I think, be deduced from a very elegant formula of $\mathbf{M}$. Borchardt (referred to in the sequel), which gives the generating function of any symmetric function of the roots, and contains potentially a method for the calculation of the Tables $(b)$, but which, from the example I have given, would not appear to be a very convenient one for actual calculation.

Suppose in general

$$
(1, b, c \ldots \gamma 1, x)^{\infty}=(1-a x)(1-\beta x)(1-\gamma x) \ldots,
$$

so that

and if in general

$$
-b=\Sigma \alpha, \quad+c=\Sigma \alpha \beta, \quad-d=\Sigma \alpha \beta \gamma, \& c
$$

$$
(p q r \ldots)=\Sigma \alpha^{p} \beta^{q} \gamma^{r} \ldots
$$

C. II. 
where as usual the summation extends only to the distinct terms, so that e.g. $\left(p^{2}\right)$ contains only half as many terms as $(p q)$, and so in all similar cases, then we have

$$
-b=(1), \quad+c=\left(1^{2}\right), \quad-d=\left(1^{3}\right), \text { \&c. }
$$

and the two problems which arise are, first to express any combination $b^{p} c^{q} \ldots$ in terms of the symmetric functions $\left(l^{x} m^{y} \ldots\right)$, and secondly, or conversely, to express any symmetric function $\left(l^{x} m^{y} \ldots\right)$ in terms of the combinations $b^{p} c^{q} \ldots$.

It will conduce materially to brevity if $1^{p} 2^{q} \ldots$ be termed the partition belonging to the combination $b^{p} c^{q} \ldots$; and in like manner if $l^{x} m^{y} \ldots$ be termed the partition belonging to the symmetric function $\left(l^{x} m^{y} \ldots\right)$, and if the sum of the component numbers of the partition is termed the weight.

Consider now a line of combinations corresponding to a given weight, e.g. the weight 4 , this will be

$$
\begin{array}{llllll}
e & b d & c^{2} & b^{2} c & b^{4} & \text { (line) } \\
4 & 13 & 2^{2} & 1^{2} 2 & 1^{4} &
\end{array}
$$

where I have written under each combination the partition which belongs to it, and in like manner a column of symmetric functions of the same weight, viz.

(4) (column)

where, as the partitions are obtained by simply omitting the ( ), I have not separately written down the partitions.

It is at once obvious that the different combinations of the line will be made up of numerical multiples of the symmetric functions of the column; and conversely, that the symmetric functions of the column will be made up of numerical multiples of the combinations of the line; but this requires a further examination. There are certain restrictions as to the symmetric functions which enter into the expression of the combination, and conversely, as to the combinations which enter into the expression of the symmetric function. The nature of the first restriction is most clearly seen by the following Table:

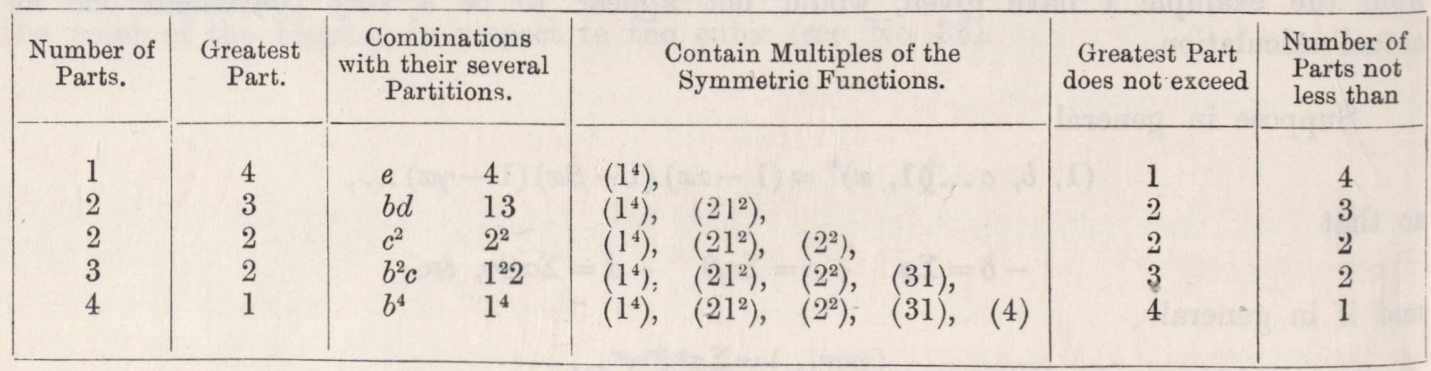


Thus, for instance, the combination $b d$ (the partition whereof is 13) contains multiples of the two symmetric functions $\left(1^{4}\right),\left(21^{2}\right)$ only. The number of parts in the partition 13 is 2 , and the greatest part is 3 . And in the partitions $\left(1^{4}\right),\left(21^{2}\right)$ the greatest part is 2, and the number of parts is not less than 3. The reason is obvious: each term of the developed expression of $b d$ must contain at least as many roots as are contained in each term of $d$, that is 3 roots, and since the coefficients are linear functions in respect to each root, the combination $b d$ cannot contain a power higher than 2 of any root. The reasoning is immediately applied to any other case, and we obtain

First Restriction.-A combination $b^{p} c^{q} \ldots$ contains only those symmetric functions $\left(l^{x} m^{y} \ldots\right)$, for which the greatest part does not exceed the number of parts in the partition $1^{p} 2^{q} \ldots$, and the number of parts is not less than the greatest part in the same partition.

Consider a partition such as $1^{2} 2$, then replacing each number by a line of units thus,

and summing the columns, we obtain a new partition 31 , which may be called the conjugate $^{1}$ of $1^{2} 2$. It is easy to see that the expression for the combination $b^{2} c$ (for which the partition is $1^{2} 2$ ) contains with the coefficient unity, the symmetric function (31), the partition whereof is the conjugate of $1^{22}$. In fact $b^{2} c=(-\Sigma \alpha)^{2}(\Sigma \alpha \beta)$, which obviously contains the term $+1 \alpha^{3} \beta$, and therefore the symmetric function with its coefficient $+1(31)$; and the reasoning is general, or

Theorem. A combination $b^{q} c^{q} \ldots$ contains the symmetric function (partition conjugate to $1^{p} 2^{q} \ldots$ ) with the coefficient unity, and sign + or - according as the weight is even or odd.

Imagine the partitions arranged as in the preceding column, viz. first the partition into one part, then the partitions into two parts, then the partitions into three parts, and so on; the partitions into the same number of parts being arranged according to the magnitude of the greatest part (the greatest magnitude first), and in case of equality according to the magnitudes of the next greatest part, and so on (for other examples, see the outside column of any one of the Tables). The order being thus completely defined, we may speak of a partition as being prior or posterior to another. We are now able to state a second restriction as follows.

Second Restriction.-The combination $b^{p} c^{q} \ldots$ contains only those symmetric functions

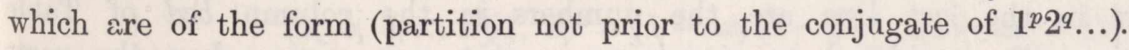

The terms excluded by the two restrictions are many of them the same, and it might at first sight appear as if the two restrictions were identical; but this is not

1 The notion of Conjugate Partitions is, I believe, due to Professor Sylvester or Mr Ferrers. [It was due to $\mathrm{Mr}$ now Dr Ferrers.] 
so: for instance, for the combination $b d^{2}$, see Table VII $(a)$, the term $\left(41^{3}\right)$ is excluded by the first restriction, but not by the second; and on the other hand, the term $\left(3^{2} 1\right)$, which is not excluded by the first restriction, is excluded by the second restriction, as containing a partition 321 prior in order to $32^{2}$, which is the partition conjugate to $13^{2}$, the partition of $b d^{2}$. It is easy to see- why $b d^{2}$ does not contain the symmetric function $\left(3^{2} 1\right)$; in fact, a term of $\left(3^{2} 1\right)$ is $\left(\alpha^{3} \beta^{3} \gamma\right)$, which is obviously not a term of $b d^{3}=(-\Sigma \alpha)(\Sigma \alpha \beta \gamma)^{2}$; but I have not investigated the general proof.

I proceed to explain the construction of the Tables $(a)$. The outside column contains the symmetric functions arranged in the order before explained; the outside or top line contains the combinations of the same weight arranged as follows, viz. the partitions taken in order from right to left are respectively conjugate to the partitions in the outside column, taken in order from top to bottom; in other words, each square of the sinister diagonal corresponds to two partitions which are conjugate to each other. It is to be noticed that the combinations taken in order, from left to right, are not in the order in which they would be obtained by Arbogast's Method of Derivations from an operand $a^{x}, a$ being ultimately replaced by unity. The squares above the sinister diagonal are empty (i.e. the coefficients are zero), the greater part of them in virtue of both restrictions, and the remainder in virtue of the second restriction; the empty squares below the sinister diagonal are empty in virtue of the second restriction; but the property was not assumed in the calculation.

The greater part of the numbers in the Tables $(a)$ were calculated, those of each table from the numbers in the next preceding table by the following method, depending on the derivation of the expression for $b^{p+1} c^{q} \ldots$ from the expression for $b^{p} c^{q} \ldots$ Suppose, for example, the column $c d$ of Table $V(a)$ is known, and we wish to calculate the column $b c d$ of Table VI $(a)$. The process is as follows:

\section{Given}

$$
\left|\begin{array}{l|l|l|}
2^{2} 1 & \frac{21^{3}}{1} & 1^{5} \\
\hline 3 & 10
\end{array}\right|
$$

we obtain

\begin{tabular}{|c|c|c|c|c|c|}
\hline 321 & $2^{3}$ & $31^{3}$ & $2^{2} 1^{2}$ & $21^{4}$ & $1^{6}$ \\
\hline 1 & 3 & 3 & $\begin{array}{l}2 \\
6\end{array}$ & $\begin{array}{l}12 \\
10\end{array}$ & 60 \\
\hline 1 & 3 & 3 & 8 & 22 & 60 \\
\hline
\end{tabular}

where the numbers in the last line are the numbers in the column $b c d$ of Table VI (a). The partition $2^{2} 1$, considered as containing a part zero, gives, when the parts are successively increased by 1 , the partitions $321,2^{3}, 2^{2} 1^{2}$, in which the indices of the increased part (i.e. the original part plus unity) are 1, 3, 2; these numbers are taken as multipliers of the coefficient 1 of the partition $2^{2} 1$, and we thus have the new coefficients $1,3,2$ of the partitions $321,2^{3}, 2^{2} 1^{2}$. In like manner the coefficient 3 of 
the partition $21^{3}$ gives the new coefficients $3,6,12$ of the partitions $31^{3}, 2^{2} 1^{2}, 21^{4}$, and the coefficient 10 of the partition $1^{5}$ gives the new coefficients 10,60 of the partitions $21^{4}$ and $1^{6}$, and finally, the last line is obtained by addition. The process in fact amounts to the multiplication separately of each term of $c d=$

$$
1\left(2^{2} 1\right)+3\left(21^{3}\right)+10\left(1^{5}\right)
$$

by $b=(1)$. It would perhaps have been proper to employ an analogous rule for the calculation of the combinations $c^{q} d^{r} \ldots$ not containing $b$, but instead of doing so I availed myself of the existing Tables $(b)$. But the comparison of the last line of each Table (a) (which as corresponding to a combination $b^{p}$ was always calculated independently of the Tables $(b)$ ) with such last line as calculated from the corresponding Table $(b)$, seems to afford a complete verification of both the Tables; and my process has in fact enabled me to detect several numerical errors in the Tables (b), as given in the English translation of the work above referred to. It is not desirable, as regards facility of calculation and independently of the want of verification, to calculate either set of Tables wholly from the other; the rules for the independent calculation of the Tables $(b)$ are fully and clearly explained in the work referred to, and I have nothing to add upon this subject.

The relation of symmetry, alluded to in the introductory paragraph of the present memoir, exists in each Table of either set, and is as follows: viz. the number in the Table corresponding to any two partitions in the outside column and the outside line respectively, is equal to the number corresponding to the same two partitions in the outside line and the outside column respectively. Or, calling the two partitions $P, Q$, and writing for shortness, combination $(P)$ for the combination represented by the partition $P$, and for greater clearness, symmetric function $(P)$ (instead of merely $(P)$ ) to denote the symmetric function represented by the partition $P$, we have the following two theorems, viz.

THEOREM. The coefficient in combination $(P)$ of symmetric function $(Q)$ is equal to the coefficient in combination $(Q)$ of symmetric function $(P)$;

and conversely,

THEorem. The coefficient in symmetric function $(P)$ of combination $(Q)$ is equal to the coefficient in symmetric function $(Q)$ of combination $(P)$.

M. Borchardt's formula, before referred to, is given in the 'Monatsbericht' of the Berlin Academy (March 5, 1885) ${ }^{1}$, and may be thus stated; viz. considering the case of $n$ roots, write

then

$$
\left(1, b, c, \ldots k^{\gamma} \gamma 1, x\right)^{n}=(1-\alpha x)(1-\beta x) \ldots(1-\kappa x)=f x,
$$

$$
\Sigma\left(\frac{1}{1-\alpha x} \frac{1}{1-\beta y} \ldots \frac{1}{1-\kappa u}\right)=\frac{1}{k}(-)^{n} \frac{f x f y \ldots f u}{\Pi(x, y, \ldots u)} \frac{d}{d x} \frac{d}{d y} \ldots \frac{d}{d u} \frac{\Pi(x, y, \ldots u)}{f x f y \ldots f u}
$$

${ }^{1}$ And in Crelle, t. liii. p. 195.-Note added 4th Dec. 1857, A. C. 
where $\Pi(x, y, \ldots u)$ denotes the product of the differences of the quantities $x, y, \ldots u$, and on the left-hand side the summation extends to all the different permutations of $\alpha, \beta, \ldots \kappa$, or what is the same thing, of $x, y, \ldots u$.

Suppose for a moment that there are only two roots, so that

$$
\left(1, b, c^{\gamma}(1, x)^{2}=(1-\alpha x)(1-\beta x),\right.
$$

then the left-hand side is

$$
\frac{1}{(1-\alpha x)(1-\beta y)}+\frac{1}{(1-\alpha y)(1-\beta x)},
$$

which is equal to

$$
2+(\alpha+\beta)(x+y)+\left(\alpha^{2}+\beta^{2}\right)\left(x^{2}+y^{2}\right)+2 \alpha \beta x y+\left(\alpha^{3}+\beta^{3}\right)\left(x^{3}+y^{3}\right)+\left(\alpha^{2} \beta+\alpha \beta^{2}\right)\left(x^{2} y+x y^{2}\right)+\& c .
$$

and the right-hand side is

$$
\text { 1: } \frac{f x f y}{x-y} \frac{d}{d x} \frac{d}{d y} \frac{x-y}{f x f y},
$$

which is equal to

$$
\frac{1}{c} \frac{f x f y}{x-y}\left\{\frac{f^{\prime} x f y-f^{\prime} y f x+(x-y) f^{\prime} x f^{\prime} y}{(f x)^{2}(f y)^{2}}\right\},
$$

and therefore to

$$
\frac{1}{c} \cdot \frac{1}{f x f y}\left\{\frac{f^{\prime} x f y-f^{\prime} y f x}{x-y}+f^{\prime} x f^{\prime} y\right\} ;
$$

or substituting for $f x, f y$ their values,

$$
\frac{f^{\prime} x f y-f^{\prime} y f x}{x-y}
$$

becomes equal to

$$
2 c-b^{2}-b c(x+y)-2 c^{2} x y,
$$

and $f^{\prime} x f^{\prime} y$ is equal to

$$
b^{2}+2 b c(x+y)+4 c^{2} x y .
$$

The right-hand side is therefore equal to

$$
\frac{2+b(x+y)+2 c x y}{\left(1+b x+c x^{2}\right)\left(1+b y+c y^{2}\right)}
$$

and comparing with the value of the left-hand side, we see that this expression may be considered as the generating function of the symmetric functions of $(\alpha, \beta)$, viz. the expression in question is developable in a series of the symmetric functions of $(x, y)$, the coefficients being of course functions of $b$ and $c$, and these coefficients are (to given numerical factors près) the symmetric functions of the roots $(\alpha, \beta)$. 
And in general it is easy to see that the left-hand side of $M$. Borchardt's formula is equal to

$$
[0]+[1](1)(1)^{\prime}+[2](2)(2)^{\prime}+\left[1^{2}\right]\left(1^{2}\right)\left(1^{2}\right)^{\prime}+\& c .
$$

where (1), (2), $\left(1^{2}\right)$, \&c. are the symmetric functions of the roots $(\alpha, \beta, \ldots \kappa),(1)^{\prime},(2)^{\prime}$, $\left(1^{2}\right)^{\prime}$, \&c. are the corresponding symmetric functions of $(x, y, \ldots u)$, and [0], [1], [2], [1 $\left.1^{2}\right]$, \&c. are mere numerical coefficients; viz. [0] is equal to $1.2 .3 \ldots n$, and [1], [2], [12], \&c. are such that the product of one of these factors into the number of terms in the corresponding symmetric function (1), (2), $\left(1^{2}\right)$, \&c. may be equal to $1.2 .3 \ldots n$. The right-hand side of $\mathbf{M}$. Borchardt's formula is therefore, as in the particular case, the generating function of the symmetric functions of the roots $(\alpha, \beta, \ldots \kappa)$, and if a convenient expression of such right-hand side could be obtained, we might by means of it express all the symmetric functions of the roots in terms of the coefficients.

\section{Tables relating to the Symmetric Functions of the Roots of an Equation.}

The outside line of letters contains the combinations (powers and products) of the coefficients, the coefficients being all with the positive sign, and the coefficient of the highest power being unity; thus in the case of a cubic equation the equation is

$$
x^{3}+b x^{2}+c x+d=(x-\alpha)(x-\beta)(x-\gamma)=0 .
$$

The outside line of numbers is obtained from that of letters merely by writing $1,2,3 \ldots$ for $b, c, d \ldots$, and may be considered simply as a different notation for the combinations. The outside column contains the different symmetric functions in the notation above explained, viz. (1) denotes $\Sigma \alpha$, (2) denotes $\Sigma \alpha^{2},\left(1^{2}\right)$ denotes $\Sigma \alpha \beta$, and so on. The Tables (a) are to be read according to the columns; thus Table II $(a)$ means $b^{2}=1(2)+2(1)^{2}$, $c=\left(1^{2}\right)$. The Tables $(b)$ are to be read according to the lines; thus Table II $(b)$ means $(2)=-2 c+1 b^{3},\left(1^{3}\right)=+1 c$.
I (a).
II (a).
III (a).
(1) \begin{tabular}{c|}
1 \\
(1) \\
\hline
\end{tabular}
\begin{tabular}{c|c|c|}
$(2)$ & $\begin{array}{c}2 \\
c\end{array}$ & $b^{2}$ \\
$\left(1^{2}\right)$ & +1 & +1 \\
\hline+2
\end{tabular}

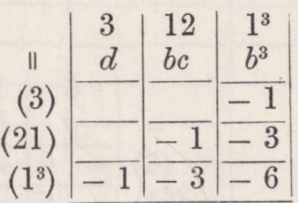
I (b).
II (b).
III (b).
\begin{tabular}{c|c|}
1 \\
(1) \\
\hline
\end{tabular}
\begin{tabular}{c|c|c}
$=$ & $c$ & $1^{2}$ \\
$(2)$ & $\frac{b^{2}}{-2}$ & +1 \\
\hline$\left(1^{2}\right)$ & +1 &
\end{tabular}

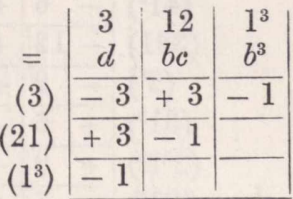


IV (a).

\begin{tabular}{|c|c|c|c|c|c|}
\hline & 4 & 13 & $2^{2}$ & $1^{2} 2$ & $1^{4}$ \\
\hline \| & $e$ & $b d$ & $c^{2}$ & $b^{2} c$ & $b^{4}$ \\
\hline (4) & & & & & +1 \\
\hline (31) & & & & +1 & +4 \\
\hline$\left(2^{2}\right)$ & & & +1 & +2 & +6 \\
\hline$\left(21^{2}\right)$ & & +1 & +2 & +5 & +12 \\
\hline$\left(1^{4}\right)$ & +1 & +4 & +6 & +12 & $\overline{+2} \overline{4}$ \\
\hline
\end{tabular}

$\mathrm{V}(a)$.

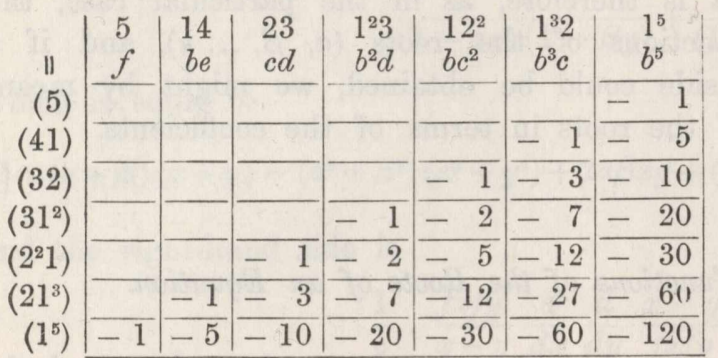

IV (b).

\begin{tabular}{|c|c|c|c|c|c|}
\hline$=$ & $\begin{array}{l}4 \\
e\end{array}$ & $\begin{array}{l}13 \\
b d\end{array}$ & $\begin{array}{l}2^{2} \\
c^{2}\end{array}$ & $\begin{array}{l}1^{22} \\
b^{2} c\end{array}$ & $\begin{array}{l}1^{4} \\
b^{4}\end{array}$ \\
\hline (4) & -4 & +4 & +2 & -4 & +1 \\
\hline (31) & +4 & -1 & -2 & +1 & \\
\hline$\left(2^{2}\right)$ & +2 & $\overline{-2}$ & +1 & & \\
\hline$\left(21^{2}\right)$ & -4 & $\overline{+1}$ & & & \\
\hline$\left(1^{4}\right)$ & +1 & & & & \\
\hline
\end{tabular}

$\mathrm{V}(b)$.

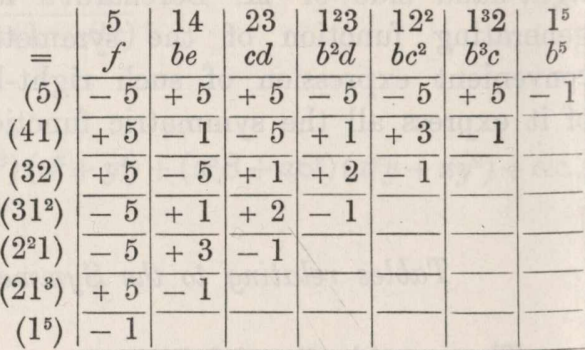

VI $(a)$.

\begin{tabular}{|c|c|c|c|c|c|c|c|c|c|c|c|}
\hline$\|$ & $\begin{array}{l}6 \\
g\end{array}$ & $\begin{array}{l}15 \\
b f\end{array}$ & $\begin{array}{l}24 \\
\mathrm{ce}\end{array}$ & $\begin{array}{l}1^{2} 4 \\
b^{2} e\end{array}$ & $\begin{array}{l}3^{2} \\
d^{2}\end{array}$ & $\begin{array}{l}123 \\
b c d\end{array}$ & $\begin{array}{l}1^{3} 3 \\
b^{3} d\end{array}$ & $\begin{array}{l}2^{3} \\
c^{3}\end{array}$ & $\begin{array}{l}1^{2} 2^{2} \\
b^{2} c^{2}\end{array}$ & $\begin{array}{l}1^{4} 2 \\
b^{4} c\end{array}$ & $\begin{array}{l}1^{6} \\
b^{6}\end{array}$ \\
\hline (6) & & & & & & & & & & & \\
\hline (51) & & & & & & & & & & & \\
\hline (42) & & & & & & & & & + & + & +15 \\
\hline$\left(3^{2}\right)$ & & & & & & & & $7 \quad 1$ & + & + & +20 \\
\hline$\left(41^{2}\right)$ & & & & & & & & $\ldots$ & + & + & +30 \\
\hline 321) & & & & & & +1 & & +3 & & $\begin{array}{r}+\quad 22 \\
\end{array}$ & \\
\hline$\left(2^{3}\right)$ & & & & & +1 & +3 & & +6 & $\begin{array}{l}+\quad 15 \\
\end{array}$ & +36 & +90 \\
\hline$\left(31^{3}\right)$ & & & & +1 & & +3 & +10 & +6 & +18 & +48 & +120 \\
\hline$\left(2^{2} 1^{2}\right)$ & & & & +2 & $\begin{array}{r}+2 \\
\end{array}$ & +8 & +18 & +15 & $\begin{array}{l}+34 \\
\end{array}$ & $\begin{array}{l}+78 \\
\end{array}$ & +180 \\
\hline & & +1 & + & +9 & +6 & +22 & +48 & +36 & $\begin{array}{l}+78 \\
\end{array}$ & +168 & +360 \\
\hline & & +6 & +15 & +30 & +20 & $\overline{+60}$ & +120 & +90 & +180 & +360 & +720 \\
\hline
\end{tabular}

VI (b).

\begin{tabular}{|c|c|c|c|c|c|c|c|c|c|c|c|}
\hline & 6 & $\begin{array}{l}15 \\
b f\end{array}$ & 24 & $\begin{array}{l}1^{2} 4 \\
b^{2} c\end{array}$ & $\begin{array}{l}3^{2} \\
d^{2}\end{array}$ & $\begin{array}{l}123 \\
b c d\end{array}$ & $\begin{array}{l}1^{3} 3 \\
h^{3} d\end{array}$ & $\begin{array}{l}2^{3} \\
c^{3}\end{array}$ & $\begin{array}{l}1^{2} 2^{2} \\
b^{2} c^{2}\end{array}$ & $\mid \begin{array}{l}1^{4} 2 \\
b^{4} c\end{array}$ & $\begin{array}{l}1^{6} \\
h^{6}\end{array}$ \\
\hline $\begin{array}{l}= \\
(6)\end{array}$ & $\frac{g}{-6}$ & $\frac{b f}{+6}$ & $\frac{c e}{+6}$ & $\frac{b^{2} c}{-6}$ & $\frac{d^{2}}{+3}$ & $\frac{b c d}{-12}$ & $\frac{b^{3} d}{+6}$ & $\frac{c^{\circ}}{-2}$ & $\frac{d c}{+9}$ & $\frac{0 c}{-6}$ & $\frac{o}{+1}$ \\
\hline 51) & +6 & $\overline{-1}$ & $\overline{-6}$ & +1 & -3 & +7 & -1 & +2 & -4 & +1 & \\
\hline 42) & 6 & -6 & $\overline{+2}$ & +2 & -3 & +4 & -2 & -2 & +1 & & \\
\hline & + & -3 & -3 & +3 & +3 & -3 & $\ldots$ & +1 & & & \\
\hline $\left.41^{2}\right)$ & -6 & +1 & $\overline{+2}$ & -1 & +3 & -3 & +1 & & & & \\
\hline 321) & -12 & +7 & +4 & $\overline{-3}$ & -3 & +1 & & & & & \\
\hline 2 & -2 & +2 & $\overline{-2}$ & $\ldots$ & +1 & & 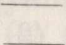 & & & & \\
\hline & +6 & -1 & $\overline{-2}$ & $\overline{+1}$ & 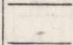 & & + & & & & \\
\hline & +9 & -4 & +1 & & & & & & & & \\
\hline & -6 & +1 & & & & & & & & & \\
\hline & + & & & & & & & & & & \\
\hline
\end{tabular}




\begin{tabular}{|c|c|c|c|c|c|c|c|c|c|c|c|c|c|c|c|c|c|}
\hline$\|$ & $\begin{array}{l}7 \\
h\end{array}$ & $\begin{array}{l}16 \\
b g\end{array}$ & $\begin{array}{l}25 \\
c f\end{array}$ & $\begin{array}{l}1^{2} 5 \\
b^{2} f\end{array}$ & $\begin{array}{l}34 \\
\text { de }\end{array}$ & $\begin{array}{r}124 \\
b c e\end{array}$ & $\begin{array}{l}1^{3} 4 \\
b^{3} e\end{array}$ & $\begin{array}{l}12^{2} \\
b d^{2}\end{array}$ & $\begin{array}{l}2^{2} 3 \\
c^{2} d\end{array}$ & $\begin{array}{l}1^{2} 23 \\
b^{2} c d\end{array}$ & $\begin{array}{l}1^{4} 3 \\
b^{4} d\end{array}$ & $\begin{array}{l}12^{3} \\
b c^{3}\end{array}$ & $\begin{array}{l}1^{3} \\
b^{3} c\end{array}$ & $\begin{array}{l}2^{2} \\
c^{2}\end{array}$ & $\begin{array}{l}1^{5} 2 \\
b^{5} c\end{array}$ & & $\begin{array}{l}1^{7} \\
b^{7}\end{array}$ \\
\hline (7) & & & & & & & & & & & & & & & & & - \\
\hline (61) & & & & & & & & & & & & & & & - & 1 & - \\
\hline (52) & & & & & & & & & & & & & - & 1 & - & 5 & - \\
\hline (43) & & & & & & & & & & & & -1 & - & 3 & - & 10 & - \\
\hline$\left(51^{2}\right)$ & & & & & & & i & & & & -1 & $\ldots$ & - & 2 & - & 11 & - \\
\hline (421) & & & & & & & & & & -1. & -4 & -3 & - & 11 & - & 35 & -105 \\
\hline$\left(3^{2} 1\right)$ & & & & & & & & & -1 & -2 & -6 & -7 & $\overline{-}$ & 18 & - & 50 & -140 \\
\hline$\left(32^{2}\right)$ & & & & & & & & -1 & -2 & -5 & -12 & -12 & - & 31 & - & 80 & -210 \\
\hline$\left(41^{3}\right)$ & & & & & & & -1 & $\ldots$ & $\ldots$ & - & -13 & -6 & - & 24 & - & 75 & -210 \\
\hline$\left(321^{2}\right)$ & & & & & & -1 & -3 & -2 & -5 & -13 & -34 & -27 & - & 68 & -1 & 70 & -420 \\
\hline$\left(2^{3} 1\right)$ & & & & & -1 & -3 & -6 & -7 & -.12 & -27 & -60 & -51 & -1 & 117 & -2 & 70 & -630 \\
\hline$\left(31^{4}\right)$ & & & & -1 & $\ldots$ & -4 & $\overline{-} 13$ & -6 & -12 & -34 & -88 & -60 & -1 & 150 & -3 & 60 & -840 \\
\hline$\left(2^{2} 1^{3}\right)$ & & & -1 & -2 & -3 & -11 & -24 & -18 & -31 & -68 & -150 & -117 & -2 & 258 & -5 & 70 & -1260 \\
\hline$\left(21^{5}\right)$ & & -1 & -5 & -11 & -10 & -35 & -75 & -50 & -80 & -170 & -360 & -270 & -5 & 570 & -12 & 00 & -2520 \\
\hline$\left(1^{7}\right)$ & -1 & -7 & $\overline{-21}$ & -42 & -35 & -105 & -210 & -140 & -210 & -420 & -840 & -630 & & 260 & -25 & 20 & -5040 \\
\hline
\end{tabular}

VII (b).

\begin{tabular}{|c|c|c|c|c|c|c|c|c|c|c|c|c|c|c|c|}
\hline$=$ & $\begin{array}{l}7 \\
h\end{array}$ & $\begin{array}{l}16 \\
b g\end{array}$ & $\begin{array}{l}25 \\
c f\end{array}$ & $\begin{array}{l}1^{2} 5 \\
b^{2} f\end{array}$ & $\begin{array}{l}34 \\
d e\end{array}$ & $\begin{array}{l}124 \\
\text { bce }\end{array}$ & $\begin{array}{l}1^{3} 4 \\
b^{3} e\end{array}$ & $\begin{array}{l}12^{2} \\
b d^{2}\end{array}$ & $\begin{array}{l}2^{2} 3 \\
c^{2} d\end{array}$ & $\begin{array}{l}1^{2} 23 \\
b^{2} c d\end{array}$ & $\begin{array}{l}1^{4} 3 \\
b^{4} d\end{array}$ & $\begin{array}{l}12^{3} \\
b c^{3}\end{array}$ & $\begin{array}{l}1^{3} 2^{2} \\
b^{3} c^{2}\end{array}$ & $\begin{array}{l}1^{5} 2 \\
b^{5} c\end{array}$ & $\begin{array}{l}1^{7} \\
b^{7}\end{array}$ \\
\hline (7) & -7 & +7 & +7 & -7 & +7 & $\overline{-14}$ & +7 & -7 & -7 & +21 & -7 & +7 & -14 & +7 & -1 \\
\hline (61) & +7 & $\overline{-1}$ & $\overline{-7}$ & +1 & -7 & +8 & -1 & +4 & +7 & -9 & +1 & -5 & +5 & $\overline{-1}$ & \\
\hline (52) & +7 & -7 & +3 & +2 & -7 & +4 & -2 & +7 & -3 & -6 & +2 & +3 & -1 & & \\
\hline (43) & +7 & -7 & -7 & +7 & +5 & +2 & -3 & -5 & +1 & +3 & $\ldots$ & -1 & & & \\
\hline$\left(51^{2}\right)$ & -7 & +1 & +2 & -1 & +7 & $\overline{-3}$ & +1 & -4 & -2 & +4 & $\overline{-1}$ & & & & \\
\hline 421) & -14 & +8 & +4 & -3 & +2 & -8 & +3 & $\overline{+1}$ & +2 & -1 & & & & & \\
\hline$\left(3^{2} 1\right)$ & -7 & +4 & +7 & -4 & -5 & +1 & $\ldots$ & +2 & $\overline{-1}$ & & & & & & \\
\hline$\left(32^{2}\right)$ & -7 & +7 & $\overline{-3}$ & -2 & +1 & +2 & $\ldots$ & $\overline{-1}$ & & & & & & & \\
\hline$\left(41^{3}\right)$ & +7 & -1 & -2 & +1 & -3 & +3 & -1 & & & & & & & & \\
\hline $\left.321^{2}\right)$ & +21 & -9 & -6 & +4 & +3 & -1 & & & & & & & & & \\
\hline$\left(2^{3} 1\right)$ & $\begin{array}{r}+\quad 7 \\
\end{array}$ & -5 & +3 & $\ldots$ & -1 & & & & & & & & & & \\
\hline$\left(31^{4}\right)$ & -7 & +1 & +2 & $\overline{-1}$ & & & & & & & & & & & \\
\hline $\left.2^{2} 1^{3}\right)$ & -14 & +5 & -1 & & & & & & & & & & & & \\
\hline$\left(21^{5}\right)$ & $\begin{array}{r}+7 \\
\end{array}$ & $\overline{-1}$ & & & & & & & & & & & & & \\
\hline$\left(1^{7}\right)$ & -1 & & & & & & & & & & & & & & \\
\hline
\end{tabular}

C. II. 
VIII (a). Runs on infrà.

\begin{tabular}{|c|c|c|c|c|c|c|c|c|c|c|c|c|c|}
\hline$\|$ & $\begin{array}{l}8 \\
i\end{array}$ & $\begin{array}{l}17 \\
b h\end{array}$ & $\begin{array}{l}26 \\
c g\end{array}$ & $\begin{array}{l}1^{26} \\
b^{2} g\end{array}$ & $\begin{array}{l}35 \\
d f\end{array}$ & $\begin{array}{c}125 \\
b c f\end{array}$ & $\begin{array}{l}1^{3} 5 \\
b^{3} f\end{array}$ & $\begin{array}{l}4^{2} \\
e^{2}\end{array}$ & $\begin{array}{l}134 \\
b d e\end{array}$ & $\begin{array}{l}2^{2} 4 \\
c^{2} e\end{array}$ & $\begin{array}{l}1^{2} 24 \\
b^{2} c e\end{array}$ & $\begin{array}{l}1^{4} 4 \\
b^{4} e\end{array}$ & $\begin{array}{l}23^{2} \\
c d^{2}\end{array}$ \\
\hline (8) & & & & & & & & & & & & & \\
\hline (71) & & & & & & & 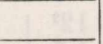 & & & & & & \\
\hline (62) & & & & & & & & & & & & & \\
\hline (53) & & & & & & & & & & & & & \\
\hline$\left(4^{2}\right)$ & & & & & & & & & & & & & \\
\hline$\left(61^{2}\right)$ & & & & & & & & & & & & & \\
\hline 521) & & & & & & & & & & & & & \\
\hline 431) & & 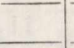 & & & & & 3 & & & & & & \\
\hline$\left(42^{2}\right)$ & & & & & & & & & & & & & \\
\hline$\left(3^{2} 2\right)$ & & & & & & & & & & & & & $+\quad 1$ \\
\hline$\left(51^{3}\right)$ & & & & & & & & & & & & & $\ldots$ \\
\hline $\left.421^{2}\right)$ & & & & & & & & & & & $+\quad 1$ & + & $\cdots$ \\
\hline$\left(3^{2} 1^{2}\right)$ & & & & & & & & & & +1 & +2 & + & \\
\hline $\left.32^{21}\right)$ & & & & & & & & & +1 & + & +5 & $+\quad 12$ & +5 \\
\hline$\left(2^{4}\right)$ & & & & & & & & +1 & +4 & +6 & +12 & +24 & +12 \\
\hline$\left(41^{4}\right)$ & & & & & & & +1 & $\ldots$ & $\ldots$ & $\ldots$ & $\begin{array}{l}+4 \\
\end{array}$ & + & $\ldots$ \\
\hline $\left.321^{3}\right)$ & & & & & & 1 & $\begin{array}{l}+3 \\
\end{array}$ & $\ldots$ & $\begin{array}{l}+\quad 3 \\
\end{array}$ & +7 & $+\quad 18$ & 46 & +12 \\
\hline$\left(2^{3} 1^{2}\right)$ & & & & & +1 & $+\quad 3$ & +6 & +2 & +11 & +18 & +39 & + & +31 \\
\hline$\left(31^{5}\right)$ & & & & +1 & $\ldots$ & +5 & +16 & $\ldots$ & +10 & +20 & +55 & +140 & +30 \\
\hline$\left(2^{2} 1^{4}\right)$ & & & +1 & +2 & +4 & +14 & +30 & +6 & +32 & +53 & $\overline{+114}$ & +246 & +80 \\
\hline$\left(21^{6}\right)$ & & +1 & +6 & $\overline{+13}$ & +15 & +51 & +108 & +20 & +95 & +150 & +315 & +660 & +210 \\
\hline$\left(1^{8}\right)$ & +1 & +8 & +28 & +56 & +56 & +168 & +336 & $|+70|$ & +280 & +420 & +840 & +1680 & +560 \\
\hline
\end{tabular}

\begin{tabular}{|c|c|c|c|c|c|c|c|c|c|}
\hline || & $\begin{array}{l}1^{2} 3^{2} \\
b^{2} d^{2}\end{array}$ & $\begin{array}{l}12^{23} \\
b c^{2} d\end{array}$ & $\begin{array}{l}l^{3} 23 \\
b^{3} c d\end{array}$ & $\begin{array}{l}1^{5} 3 \\
b^{5} d\end{array}$ & $\begin{array}{l}2^{4} \\
c^{4}\end{array}$ & $\begin{array}{l}1^{2} 2^{3} \\
b^{2} c^{3}\end{array}$ & $\begin{array}{l}1^{4} 2^{2} \\
b^{4} c^{2}\end{array}$ & $\begin{array}{l}1^{62} \\
b^{6} c\end{array}$ & $\begin{array}{l}1^{8} \\
b^{8}\end{array}$ \\
\hline (8) & & & & & & & & & + \\
\hline 1) & & & & & & & & + & + \\
\hline & & & & & & & 1 & 6 & 28 \\
\hline & & & & & & & 4 & 15 & 56 \\
\hline & & & & & & + & 6 & 20 & 70 \\
\hline & & & & 1 & $\ldots$ & $\ldots$ & + & 13 & 56 \\
\hline & & & 1 & 5 & $\ldots$ & + & 14 & 51 & 68 \\
\hline & & 1 & 3 & 10 & 4 & 11 & $\overline{32}$ & 95 & 280 \\
\hline & 1 & 2 & 7 & 20 & & & 53 & 150 & 420 \\
\hline & 2 & 5 & 12 & 30 & $+\quad 12$ & 31 & 80 & 210 & 560 \\
\hline & $\ldots$ & $\ldots$ & 3 & 16 & & + & 30 & 108 & 336 \\
\hline & $+\quad 2$ & + & 18 & 55 & $+\quad 12$ & & 114 & 315 & 840 \\
\hline & & & 30 & 80 & & & 172 & 440 & 1120 \\
\hline & 12 & 24 & 58 & +140 & 48 & +117 & 284 & 690 & 1680 \\
\hline & 28 & 48 & 108 & +240 & & +204 & 468 & +1080 & 2520 \\
\hline & & 12 & & +140 & & +84 & 246 & & 1680 \\
\hline & 30 & 58 & 141 & +340 & +108 & +258 & $+\quad 612$ & +1440 & 360 \\
\hline & 68 & 117 & 258 & +570 & +204 & +453 & +1008 & +2250 & 5040 \\
\hline & +80 & +140 & 340 & +800 & +240 & +570 & +1320 & +3000 & +6720 \\
\hline & 172 & & 612 & +1320 & +468 & +1008 & +2172 & +4680 & +10080 \\
\hline & 440 & & $\overline{1440}$ & $+30 \overline{00}$ & +1080 & +2250 & +4680 & 9720 & 160 \\
\hline & +1120 & +1680 & +3360 & +6720 & +2520 & +5040 & +10080 & +20160 & +40320 \\
\hline
\end{tabular}


VIII $(b)$. Runs on infrà.

\begin{tabular}{|c|c|c|c|c|c|c|c|c|c|c|c|c|c|}
\hline$=$ & $\begin{array}{l}8 \\
i\end{array}$ & $\begin{array}{l}17 \\
b /\end{array}$ & $\begin{array}{l}26 \\
c g\end{array}$ & $\begin{array}{l}1^{2} 6 \\
b^{2} g\end{array}$ & $\begin{array}{l}35 \\
d f\end{array}$ & $\begin{array}{l}125 \\
b c f\end{array}$ & $\begin{array}{l}1^{3} 5 \\
b^{3} f\end{array}$ & $\begin{array}{l}4^{2} \\
e^{2}\end{array}$ & $\begin{array}{l}134 \\
b d e\end{array}$ & $\begin{array}{l}2^{2} 4 \\
c^{2} e\end{array}$ & $\begin{array}{l}1^{22} 24 \\
b^{2} c e\end{array}$ & $\begin{array}{l}1^{4} 4 \\
b^{4} e\end{array}$ & $\begin{array}{l}23^{2} \\
c d^{2}\end{array}$ \\
\hline (8) & -8 & +8 & +8 & -8 & +8 & -16 & +8 & $\overline{+4}$ & -16 & -8 & +24 & -8 & -8 \\
\hline (71) & & -1 & - & +1 & -8 & +9 & -1 & $\overline{-4}$ & +9 & +8 & -10 & +1 & +8 \\
\hline (62) & 8 & - & + & +2 & -8 & +4 & -2 & $\overline{-4}$ & +16 & -4 & -6 & +2 & +2 \\
\hline (53) & +8 & - & -8 & +8 & +7 & +1 & -3 & $\overline{-4}$ & +1 & +8 & -9 & +3 & -7 \\
\hline$\left(4^{2}\right)$ & +4 & - & $=$ & +4 & -4 & +8 & -4 & +6 & -8 & -4 & +4 & $\ldots$ & +4 \\
\hline$\left(61^{2}\right)$ & -8 & + & + & -1 & +8 & -3 & +1 & $\overline{+4}$ & -9 & $\overline{-2}$ & +4 & -1 & -5 \\
\hline$(521)$ & -16 & + & +4 & -3 & +1 & -8 & +3 & +8 & -10 & $\overline{-4}$ & +11 & -3 & +5 \\
\hline (431) & -16 & + & +16 & -9 & +1 & -10 & +4 & -8 & +10 & $\ldots$ & -1 & $\ldots$ & -1 \\
\hline$\left(42^{2}\right)$ & -8 & & -4 & -2 & +8 & -4 & +2 & $\overline{-4}$ & $\ldots$ & +4 & -2 & $\ldots$ & -2 \\
\hline$\left(3^{22}\right)$ & -8 & + & +2 & -5 & -7 & +5 & $\ldots$ & +4 & -1 & -2 & $\ldots$ & $\ldots$ & +1 \\
\hline$\left(51^{3}\right)$ & +8 & -1 & -2 & +1 & -3 & +3 & -1 & $\overline{-4}$ & +4 & +2 & -4 & +1 & \\
\hline $\left.421^{2}\right)$ & +24 & -10 & -6 & +4 & -9 & +11 & -4 & +4 & -1 & -2 & +1 & & \\
\hline & +12 & -5 & -9 & +5 & +3 & -1 & $\ldots$ & +2 & -2 & +1 & & & \\
\hline $\left.32^{2} 1\right)$ & +24 & -17 & $\ldots$ & +5 & +6 & -3 & $\ldots$ & $\overline{-4}$ & +1 & & & & \\
\hline$\left(2^{4}\right)$ & +2 & -2 & +2 & $\ldots$ & -2 & 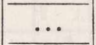 & $\ldots$ & +1 & & & & & \\
\hline$\left(41^{4}\right)$ & -8 & +1 & + & -1 & +3 & -3 & +1 & & & & & & \\
\hline $321^{3}$ & -32 & +11 & +8 & -5 & -3 & +1 & & & & & & & \\
\hline & -16 & +9 & -4 & $\ldots$ & +1 & & & & & & & & \\
\hline$\left(31^{5}\right.$ & +8 & -1 & -2 & +1 & & & & * & & & & & \\
\hline$\left(2^{2} 1^{4}\right)$ & +20 & -6 & +1 & & & & & & & & & & \\
\hline (21 & $\begin{array}{r}-8 \\
\end{array}$ & +1 & & & & & & & & & & & \\
\hline & +1 & & & & & & & & & & & & \\
\hline
\end{tabular}

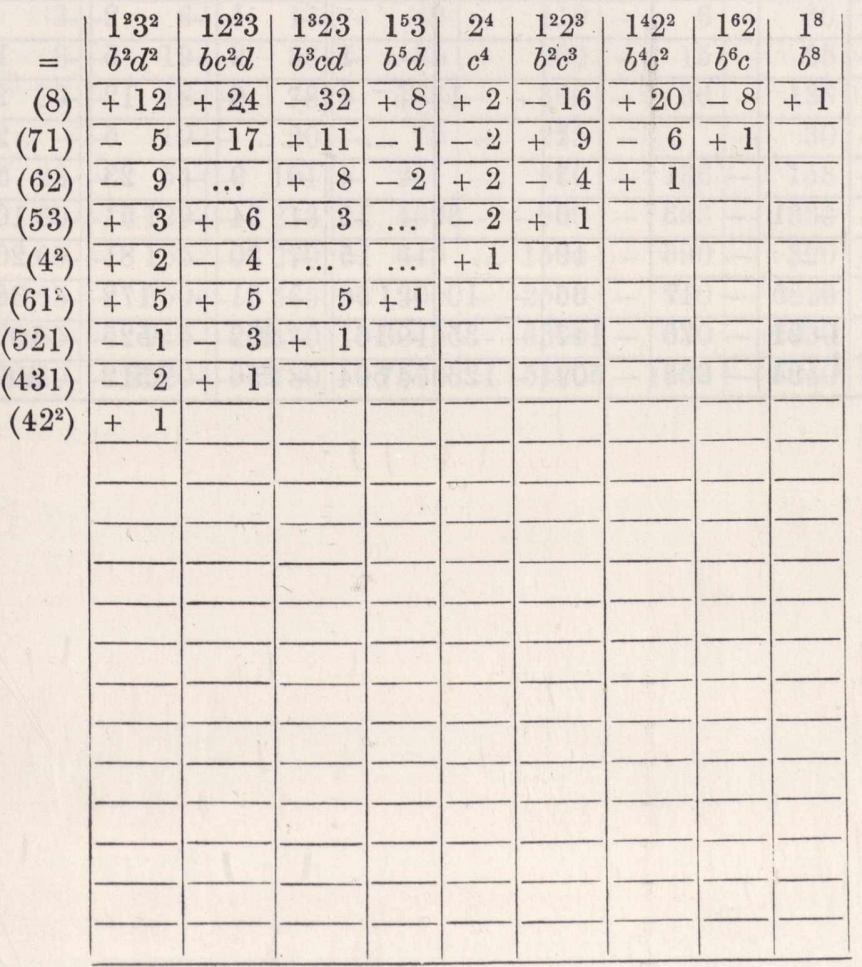


IX (a). Runs on to p. 430 .

\begin{tabular}{|c|c|c|c|c|c|c|c|c|c|c|c|c|c|}
\hline$\|$ & $\begin{array}{l}9 \\
j\end{array}$ & $\begin{array}{l}18 \\
b i\end{array}$ & $\begin{array}{l}2 \bar{\lambda} \\
c h\end{array}$ & $\begin{array}{l}1^{2} 7 \\
b^{2} h\end{array}$ & $\begin{array}{l}35 \\
d g\end{array}$ & $\begin{array}{l}125 \\
b c g\end{array}$ & $\begin{array}{l}1^{3} 6 \\
b^{3} g \\
\end{array}$ & $\begin{array}{l}45 \\
e f\end{array}$ & $\begin{array}{l}135 \\
b d f\end{array}$ & $\begin{array}{l}2^{25} \\
c^{2} f\end{array}$ & $\begin{array}{l}1^{2} 25 \\
b^{2} c f \\
\end{array}$ & $\begin{array}{l}1^{4} 5 \\
b^{4} f\end{array}$ & $\begin{array}{l}14^{2} \\
b e^{2}\end{array}$ \\
\hline (9) & & 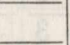 & & & th & & 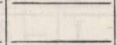 & & & & & 80 & \\
\hline (81) & & & & & & & & & & & & $\theta$ & \\
\hline (72) & & & & & - & 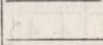 & 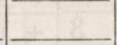 & _ & & 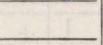 & & & \\
\hline (63) & & & 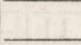 & & Z & & 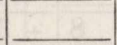 & $\square$ & & 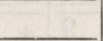 & - & 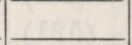 & \\
\hline (54) & & & ne & & $\theta$ & & 38 & & & & & 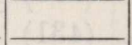 & \\
\hline$\left(71^{2}\right)$ & & & & & & & $\square$ & & & & & +2 & \\
\hline (621) & & & . & 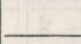 & 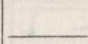 & 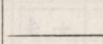 & 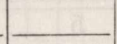 & & & & 3 & $\ldots$ & \\
\hline (531) & & L & L & & & & & & & & & 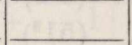 & \\
\hline$\left(4^{2} 1\right)$ & & & - & & & & 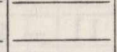 & & & & & & \\
\hline$\left(52^{2}\right)$ & & 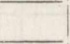 & 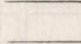 & 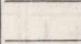 & 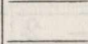 & $\square$ & - & & & & & $x$ & \\
\hline (432) & & E & 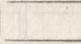 & & 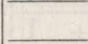 & & 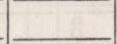 & & & & & 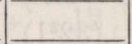 & \\
\hline$\left(3^{3}\right)$ & & & 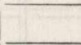 & & & 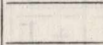 & & & & & & & \\
\hline$\left(61^{3}\right)$ & & & & & & & + & & & 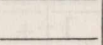 & 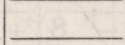 & $4=$ & \\
\hline$\left(521^{2}\right)$ & & $E$ & E & & & & 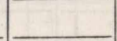 & & & r & Ba & 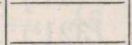 & \\
\hline$\left(431^{2}\right)$ & & & & & & & & & & & & & \\
\hline$\left(42^{2} 1\right)$ & & & E & & & & & & & & & & \\
\hline$\left(3^{2} 21\right)$ & & & 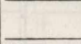 & & & & & & & & 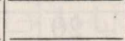 & 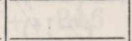 & \\
\hline$\left(32^{3}\right)$ & & & 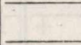 & & & & & & & & & & -1 \\
\hline$\left(51^{4}\right)$ & & & & & & & & & & & & - & $\ldots$ \\
\hline$\left(421^{3}\right)$ & & & & & & & & & & & -1 & - & $\ldots$ \\
\hline$\left(3^{2} 1^{3}\right)$ & & & & & & & & & & - & - & - & \\
\hline$\left(32^{2} 1^{2}\right)$ & & & & & & & & & -1 & - & - & - & \\
\hline$\left(2^{4} 1\right)$ & & & & & $t$ & & $y=$ & -1 & -4 & -6 & -12 & -24 & -9 \\
\hline$\left(41^{5}\right)$ & & & & & & & -1 & $\ldots$ & $\ldots$ & $\ldots$ & -5 & -21 & $\ldots$ \\
\hline$\left(321^{4}\right)$ & & & & & & - & -3 & $\ldots$ & -4 & -9 & -23 & -58 & -6 \\
\hline$\left(2^{3} 1^{3}\right)$ & & & & & -1 & -3 & -6 & -3 & -15 & -24 & -51 & -108 & -24 \\
\hline$\left(31^{6}\right)$ & & & & -1 & $\ldots$ & -6 & -19 & $\ldots$ & -15 & -30 & -81 & -204 & -20 \\
\hline$\left(2^{2} 1^{5}\right)$ & & & -1 & -2 & -5 & -17 & -36 & -10 & -50 & -81 & -172 & -366 & -70 \\
\hline$\left(21^{7}\right)$ & & -1 & -7 & -15 & -21 & -70 & -147 & -35 & -161 & -252 & -525 & -1092 & -210 \\
\hline$\left(1^{9}\right)$ & -1 & $\overline{-9}$ & -36 & -72 & -84 & -252 & -504 & -126 & -504 & -756 & -1512 & -3024 & -630 \\
\hline
\end{tabular}




\begin{tabular}{|c|c|c|c|c|c|c|c|c|c|c|}
\hline II & $\begin{array}{l}234 \\
\text { cde }\end{array}$ & $\begin{array}{l}1^{2} 34 \\
b^{2} d e\end{array}$ & $\begin{array}{l}12^{2} 4 \\
b c^{2} e\end{array}$ & $\begin{array}{l}1^{3} 24 \\
b^{3} c e\end{array}$ & $\begin{array}{l}1^{5} 4 \\
b^{5} e\end{array}$ & $\begin{array}{l}3^{3} \\
d^{3}\end{array}$ & $\begin{array}{l}123^{2} \\
b c d^{2}\end{array}$ & $\begin{array}{l}1^{3} 3^{2} \\
b^{3} d^{2}\end{array}$ & $\begin{array}{l}2^{3} 3 \\
c^{3} d\end{array}$ & $\begin{array}{l}1^{2} 2^{2} 3 \\
b^{2} c^{2} d\end{array}$ \\
\hline (9) & 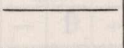 & & & & & & & & & \\
\hline (81) & 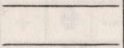 & & & & & & & & & \\
\hline (72) & $c_{2}$ & & & & & & & & & \\
\hline (63) & 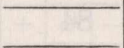 & & 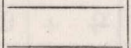 & & & & & & & \\
\hline (54) & & & 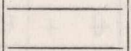 & 4 & & & & & & \\
\hline$\left(71^{2}\right)$ & -7 & & 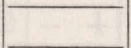 & & & & ? & & & \\
\hline (621) & $=$ & & & & 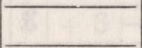 & & - & & & \\
\hline (531) & t & & & 7 & 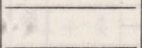 & + & 4 & & & - \\
\hline$\left(4^{2} 1\right)$ & 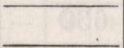 & & & 7 & 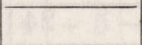 & 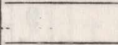 & 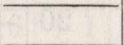 & & -1 & - \\
\hline$\left(52^{2}\right)$ & 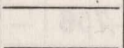 & & & & 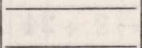 & + & 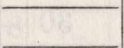 & - & $\ldots$ & - \\
\hline$(432)$ & $\theta$ & & & & & & $-\quad 1$ & - & $-\quad 3$ & - \\
\hline$\left(3^{3}\right)$ & 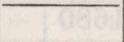 & & & & & -1 & -3 & - & -6 & - \\
\hline$\left(61^{3}\right)$ & 1 & & & r & - & $\ldots$ & $\ldots$ & $\ldots$ & $\ldots$ & $\ldots$ \\
\hline$\left(521^{2}\right)$ & 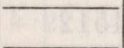 & & & - & - & $\ldots$ & $\ldots$ & - & $\ldots$ & - \\
\hline$\left(431^{2}\right)$ & 6 & & - & - & 10 & $\ldots$ & -2 & - & $\begin{array}{l}-7 \\
\end{array}$ & - \\
\hline$\left(42^{2} 1\right)$ & & - & - & 7 & 20 & $\ldots$ & -5 & 17 & $\begin{array}{l}-12 \\
\end{array}$ & - \\
\hline$\left(3^{2} 21\right)$ & -1 & 2 & - & $-\quad 12$ & 30 & $\begin{array}{l}-\quad 3 \\
\end{array}$ & -13 & 30 & -27 & - \\
\hline$\left(32^{3}\right)$ & $\begin{array}{l}-\quad 3 \\
\end{array}$ & -7 & $-\quad 12$ & -27 & 60 & -6 & $\begin{array}{l}-27 \\
\end{array}$ & 64 & -51 & 120 \\
\hline$\left(51^{4}\right)$ & $\ldots$ & $\ldots$ & $\ldots$ & $-\quad 4$ & - & $\ldots$ & $\ldots$ & - & $\ldots$ & - \\
\hline$\left(421^{3}\right)$ & $\ldots$ & $\begin{array}{l}-\quad 3 \\
\end{array}$ & -7 & -.25 & 75 & $\ldots$ & -12 & 42 & -27 & - \\
\hline$\left(3^{2} 1^{3}\right)$ & -3 & -6 & 17 & -42 & 110 & -6 & $-\quad 30$ & 72 & -64 & 152 \\
\hline $\left.32^{2} 1^{2}\right)$ & -88 & $-\quad 19$ & $\begin{array}{l}-36 \\
\end{array}$ & $-\quad 85$ & 200 & $-\quad 15$ & -65 & $-\quad 152$ & -120 & 281 \\
\hline$\left(2^{4} 1\right)$ & -22 & $-\quad 48$ & $7-78$ & -168 & 360 & $\begin{array}{l}-\quad 36 \\
\end{array}$ & -136 & $-\quad 300$ & -234 & 516 \\
\hline$\left(41^{5}\right)$ & $\ldots$ & -10 & -20 & -75 & $-\quad 225$ & $\ldots$ & -30 & $-\quad 110$ & -60 & 200 \\
\hline$\left(321^{4}\right)$ & $-\quad 22$ & -54 & -101 & -241 & $-\quad 570$ & $-\quad 36$ & -158 & 372 & -282 & $-\quad 656$ \\
\hline$\left(2^{3} 1^{3}\right)$ & -60 & -129 & -213 & -459 & $-\quad 990$ & $\begin{array}{l} \\
-\quad 93 \\
\end{array}$ & -333 & 720 & -555 & -1203 \\
\hline$\left(31^{6}\right)$ & $\div \quad 60$ & -155 & -270 & -645 & -1500 & $\begin{array}{l}-90 \\
-\quad 9\end{array}$ & -390 & -920 & -660 & -1530 \\
\hline$\left(2^{2} 1^{5}\right)$ & -165 & -350 & -565 & -1200 & -2550 & -240 & -820 & -1740 & -1320 & -2800 \\
\hline$\left(21^{7}\right)$ & -455 & -945 & -1470 & -3045 & -6300 & -630 & -2030 & -4200 & -3150 & -6510 \\
\hline$\left(1^{9}\right)$ & -1260 & -2520 & -3780 & -7560 & -15120 & -1680 & -5040 & -10080 & -7560 & -15120 \\
\hline
\end{tabular}




\begin{tabular}{|c|c|c|c|c|c|c|c|c|c|c|c|c|c|c|}
\hline$\|$ & & $\begin{array}{l}1^{4} 23 \\
b^{4} c d\end{array}$ & & $\begin{array}{l}1^{6} 3 \\
b^{6} d\end{array}$ & & $\begin{array}{l}12^{4} \\
b c^{4}\end{array}$ & & $\begin{array}{l}1^{3} 2^{3} \\
b^{3} c^{3}\end{array}$ & & $\begin{array}{l}1^{5} 2^{2} \\
b^{5} c^{2}\end{array}$ & & $\begin{array}{l}1^{7} 2 \\
b^{7} c\end{array}$ & & $\begin{array}{l}1^{9} \\
b^{9}\end{array}$ \\
\hline (9) & & & & & & & & & & & & & $=$ & 1 \\
\hline (81) & & & & & & & & & & & - & 1 & - & 9 \\
\hline (72) & & & & & & & & & - & 1 & - & 7 & $=$ & 36 \\
\hline (63) & & & & & & & - & 1 & - & 5 & - & 21 & - & 84 \\
\hline (54) & & & & & - & 1 & - & 3 & 一 & 10 & - & 35 & - & 126 \\
\hline$\left(71^{2}\right)$ & & & - & 1 & & $\ldots$ & & $\ldots$ & - & 2 & - & 15 & ב & 72 \\
\hline (621) & - & 1 & - & 6 & & $\ldots$ & - & 3 & - & 17 & - & 70 & - & 252 \\
\hline (531) & $\overline{-}$ & 4 & - & 15 & - & 4 & - & 15 & - & 50 & - & 161 & ב & 504 \\
\hline$\left(4^{2} 1\right)$ & - & 6 & - & 20 & - & 9 & - & 24 & - & 70 & - & 210 & - & 630 \\
\hline$\left(52^{2}\right)$ & - & $\overline{9}$ & - & 30 & - & 6 & - & 24 & - & $\overline{81}$ & - & 252 & - & 756 \\
\hline (432) & - & 22 & - & 60 & - & 22 & - & 60 & - & 165 & - & 455 & - & 1260 \\
\hline$\left(3^{3}\right)$ & - & 36 & - & 90 & - & 36 & ב & 93 & - & 240 & - & 630 & - & 1680 \\
\hline$\left(61^{3}\right)$ & - & 3 & - & $\overline{19}$ & & $\ldots$ & - & 6 & - & 36 & - & 147 & - & 504 \\
\hline$\left(521^{2}\right)$ & - & $\overline{23}$ & - & 81 & - & 12 & - & 51 & - & 172 & - & 525 & - & 1512 \\
\hline$\left(431^{2}\right)$ & - & 54 & - & 155 & - & 48 & - & 129 & - & 350 & - & 945 & - & 2520 \\
\hline$\left(42^{2} 1\right)$ & - & 101 & 一 & 270 & - & 78 & $=$ & 213 & - & 565 & - & 1470 & - & 3780 \\
\hline$\left(3^{2} 21\right)$ & - & 158 & - & 390 & $\overline{-}$ & 136 & $\bar{Z}$ & 333 & - & 820 & - & 2030 & ב & 5040 \\
\hline$\left(32^{3}\right)$ & - & 282 & - & 660 & $\overline{-}$ & 234 & $\overline{-}$ & 555 & 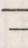 & 1320 & - & 3150 & ב & 7560 \\
\hline$\left(51^{4}\right)$ & - & 58 & - & 204 & - & 24 & - & 108 & - & 366 & - & 1092 & - & 3024 \\
\hline$\left(421^{3}\right)$ & - & 241 & - & 645 & - & 168 & Z & 459 & - & 1200 & 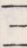 & 3045 & - & 7560 \\
\hline$\left(3^{2} 1^{3}\right)$ & & 372 & - & 920 & - & 300 & 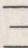 & 720 & - & 1740 & - & 4200 & - & 10080 \\
\hline$\left(32^{2} 1^{2}\right)$ & & 656 & - & 1530 & - & 516 & - & 1203 & & 2800 & - & 6510 & & 15120 \\
\hline$\left(2^{4} 1\right)$ & & 1140 & - & 2520 & - & 906 & - & 2016 & - & 4500 & - & 10080 & - & 22680 \\
\hline$\left(41^{5}\right)$ & & 570 & - & 1500 & & 360 & & 990 & 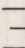 & 2550 & - & 6300 & - & 15120 \\
\hline$\left(321^{4}\right)$ & & 1516 & - & 3480 & & -1140 & & 2610 & - & 5940 & - & 13440 & - & 30240 \\
\hline$\left(2^{3} 1^{3}\right)$ & & 2610 & - & 5670 & & 2016 & - & 4383 & & 9540 & - & 20790 & - & 45360 \\
\hline$\left(31^{6}\right)$ & & 3480 & & 7800 & & -2520 & & 5670 & & 12600 & & 27720 & & 60480 \\
\hline$\left(2^{2} 1^{5}\right)$ & & 5940 & & 12600 & & 4500 & & 9540 & & 20220 & & 42840 & & 90720 \\
\hline$\left(21^{7}\right)$ & & 13440 & & 27720 & & -10080 & & 20790 & & 42840 & & 88200 & & 181440 \\
\hline$\left(1^{9}\right)$ & & 30240 & & $\overline{60480}$ & & -22680 & & 45360 & & 90720 & & 181440 & & 362880 \\
\hline
\end{tabular}


IX (b). Runs on to p. 432 .

\begin{tabular}{|c|c|c|c|c|c|c|c|c|c|c|c|c|c|c|c|}
\hline$=$ & $\begin{array}{l}9 \\
j\end{array}$ & $\begin{array}{l}18 \\
b i\end{array}$ & $\begin{array}{l}27 \\
\text { ch }\end{array}$ & $\begin{array}{l}1^{2} 7 \\
b^{2} h\end{array}$ & $\begin{array}{l}35 \\
d g\end{array}$ & $\begin{array}{l}125 \\
b c g\end{array}$ & $\begin{array}{l}1^{3} 6 \\
b^{3} g\end{array}$ & $\begin{array}{l}45 \\
e f\end{array}$ & $\begin{array}{l}135 \\
b d f\end{array}$ & $\begin{array}{l}2^{2} 5 \\
c^{2} f\end{array}$ & $\begin{array}{l}1^{2} 25 \\
b^{2} c f\end{array}$ & $\begin{array}{l}1^{4} 5 \\
b^{4} f\end{array}$ & $\begin{array}{l}14^{2} \\
b e^{2}\end{array}$ & $\begin{array}{l}234 \\
\text { cde }\end{array}$ & $\begin{array}{l}1^{2} 34 \\
b^{2} d e\end{array}$ \\
\hline (9) & -9 & +9 & +9 & -9 & $\begin{array}{l}+9 \\
\end{array}$ & -18 & +9 & +9 & -18 & $\overline{-9}$ & +27 & -9 & -9 & $\overline{-18}$ & +27 \\
\hline (81) & +9 & -1 & -9 & 1 & -9 & +10 & -1 & $\begin{array}{l}-9 \\
\end{array}$ & +10 & $\overline{+9}$ & -11 & +1 & + & $\overline{+18}$ & -11 \\
\hline (72) & + & -9 & $\begin{array}{r}+5 \\
\end{array}$ & 2 & -9 & +4 & -2 & -9 & +18 & -5 & -6 & +2 & + & +4 & -20 \\
\hline (63) & $\begin{array}{l}+9 \\
\end{array}$ & $\begin{array}{l}-9 \\
-\quad 9\end{array}$ & - & 9 & $\begin{array}{l}+\quad 9 \\
+\end{array}$ & $\ldots$ & -3 & -9 & $\ldots$ & +9 & -9 & +3 & +9 & $\bar{\ldots}$ & -9 \\
\hline (54) & +9 & -9 & -9 & $\begin{array}{l}+\quad 9 \\
\end{array}$ & -9 & +18 & -9 & +11 & -2 & $\overline{-1}$ & - & +4 & -11 & -2 & +13 \\
\hline$\left(71^{2}\right)$ & -9 & +1 & 2 & -1 & $\begin{array}{l}+\quad 9 \\
+\end{array}$ & -3 & +1 & +9 & -10 & $\overline{-2}$ & +4 & -1 & -5 & -11 & +11 \\
\hline (621) & -18 & +10 & +4 & -3 & $\ldots$ & -8 & +3 & +18 & -10 & $\overline{-4}$ & +11 & $\overline{-3}$ & -14 & $\overline{-4}$ & +13 \\
\hline (531) & -18 & +10 & +18 & -10 & $\ldots$ & -10 & +4 & -2 & -5 & -8 & +15 & $\overline{-4}$ & +6 & + & -5 \\
\hline$\left(4^{2} 1\right)$ & -9 & +5 & $\begin{array}{r}+\quad 9 \\
\end{array}$ & -5 & $\begin{array}{r}+\quad 9 \\
\end{array}$ & -14 & +5 & -11 & $\begin{array}{l}+6 \\
\end{array}$ & +1 & -1 & $\ldots$ & + & + & - \\
\hline$\left(52^{2}\right)$ & -9 & +9 & -5 & -2 & +9 & -4 & +2 & -1 & -8 & $\ldots$ & +6 & $\overline{-2}$ & + & + & $\ldots$ \\
\hline (432) & -18 & +18 & $\begin{array}{r}+\quad 4 \\
\end{array}$ & -11 & $\ldots$ & -4 & +5 & -2 & $\begin{array}{l}+\quad 2 \\
\end{array}$ & +6 & -5 & $\ldots$ & + & - & +1 \\
\hline$\left(3^{3}\right)$ & -3 & $\begin{array}{l}+\quad 3 \\
\end{array}$ & $\begin{array}{l}+\quad 3 \\
\end{array}$ & -3 & -6 & +3 & $\ldots$ & +3 & + & $\overline{-3}$ & $\ldots$ & $\ldots$ & - & + & $\ldots$ \\
\hline$\left(61^{3}\right)$ & $\begin{array}{l}+\quad 9 \\
+\end{array}$ & -1 & -2 & +1 & $\begin{array}{l}-3 \\
\end{array}$ & $\begin{array}{l}+3 \\
+\quad 3\end{array}$ & -1 & -9 & +4 & +2 & -4 & +1 & + & + & -5 \\
\hline$\left(521^{2}\right)$ & +27 & -11 & -6 & $\begin{array}{l}+4 \\
+\quad 4\end{array}$ & -9 & +11 & -4 & -7 & +15 & +6 & -15 & +4 & - & - & + \\
\hline$\left(431^{2}\right)$ & +27 & -11 & $\overline{-20}$ & +11 & -9 & +13 & $\overline{-5}$ & +13 & -5 & $\bar{\ldots}$ & +1 & $\ldots$ & - & + & + \\
\hline$\left(42^{2} 1\right)$ & +27 & -19 & +1 & +5 & -9 & +12 & -5 & $\begin{array}{r}+3 \\
\end{array}$ & -2 & $\overline{-6}$ & $\begin{array}{l}+3 \\
+\quad \\
\end{array}$ & $\ldots$ & + & + & - \\
\hline$\left(3^{2} 21\right)$ & +27 & -19 & -13 & +12 & +18 & -7 & $\ldots$ & - & -4 & $\overline{+3}$ & $\ldots$ & $\ldots$ & + & - & \\
\hline$\left(32^{3}\right)$ & $\begin{array}{l}+9 \\
\end{array}$ & -9 & +5 & $\begin{array}{l}+\quad 2 \\
\end{array}$ & -3 & -2 & $\ldots$ & +1 & + & $\ldots$ & $\ldots$ & $\ldots$ & -1 & & \\
\hline$\left(51^{4}\right)$ & -9 & +1 & +2 & -1 & +3 & -3 & +1 & + & -4 & -2 & +4 & $\overline{-1}$ & & & \\
\hline$\left(421^{3}\right)$ & -36 & +12 & +8 & -5 & +12 & -14 & +5 & -4 & & +2 & -1 & & & & \\
\hline$\left(3^{2} 1^{3}\right)$ & -18 & $\begin{array}{l}+6 \\
+\quad 6\end{array}$ & +11 & -6 & -3 & +1 & $\ldots$ & -2 & $\begin{array}{l}+2 \\
+\quad 2\end{array}$ & $\overline{-1}$ & & & & & \\
\hline $32^{2} 1^{2}$ ) & $\overline{-54}$ & +30 & +5 & -9 & $\begin{array}{l}-9 \\
\end{array}$ & +4 & $\ldots$ & $+\quad 4$ & -1 & & & & & & \\
\hline$\left(2^{4} 1\right)$ & $\begin{array}{l}-9 \\
\end{array}$ & $\begin{array}{l}+7 \\
+\quad 7\end{array}$ & -5 & $\ldots$ & $\begin{array}{l}+3 \\
\end{array}$ & $\ldots$ & $\ldots$ & -1 & & & & & & & \\
\hline$\left(41^{5}\right)$ & +9 & -1 & -2 & +1 & -3 & +3 & -1 & & & & & & & & \\
\hline$\left(321^{4}\right)$ & +45 & -13 & -10 & +6 & +3 & -1 & & & & & & & & & \\
\hline$\left(2^{3} 1^{3}\right)$ & +30 & -14 & +5 & $\ldots$ & -1 & & & & & & & & & & \\
\hline$\left(31^{6}\right)$ & $\div \quad 9$ & +1 & $\begin{array}{l}+\quad 2 \\
\end{array}$ & -1 & & & & & & & & & & & \\
\hline$\left(2^{2} 1^{5}\right)$ & -27 & +7 & -1 & & & & & & & & & & & & \\
\hline$\left(21^{7}\right)$ & $\begin{array}{l}+\quad 9 \\
\end{array}$ & -1 & & & & & & & & & & & & & \\
\hline$\left(1^{9}\right)$ & -1 & & & & & & & & & & & & & & \\
\hline
\end{tabular}




\begin{tabular}{|c|c|c|c|c|c|c|c|c|c|c|c|c|c|c|c|}
\hline$=$ & $\begin{array}{l}12^{2} 4 \\
b c^{2} e\end{array}$ & $\begin{array}{l}1^{3} 24 \\
b^{3} c e\end{array}$ & $\begin{array}{l}1^{5} 4 \\
b^{5} e\end{array}$ & $\begin{array}{c}3^{3} \\
d^{3}\end{array}$ & $\begin{array}{l}123^{2} \\
b c d^{2}\end{array}$ & $\begin{array}{l}1^{3} 3^{3} \\
b^{3} d^{2}\end{array}$ & $\begin{array}{l}2^{3} 3 \\
c^{3} d\end{array}$ & $\begin{array}{l}1^{2} 2^{2} 3 \\
b^{2} c^{2} d\end{array}$ & $\begin{array}{l}1^{4} 23 \\
b^{4} c d\end{array}$ & $\begin{array}{l}1^{6} 3 \\
b^{6} d\end{array}$ & $\begin{array}{l}12^{4} \\
b c^{4}\end{array}$ & $\begin{array}{l}1^{3} 2^{3} \\
b^{3} c^{3}\end{array}$ & $\begin{array}{l}1^{5} 2^{2} \\
b^{5} c^{2}\end{array}$ & $\begin{array}{l}1^{72} \\
b^{7} c\end{array}$ & $\begin{array}{l}1^{9} \\
b^{9}\end{array}$ \\
\hline (9) & +27 & $\overline{-36}$ & +9 & $\overline{-3}$ & +27 & -18 & +9 & -54 & +45 & -9 & $\overline{-9}$ & +30 & -27 & +9 & -1 \\
\hline (81) & -19 & +12 & -1 & $\overline{+3}$ & -19 & +6 & -9 & +30 & -13 & +1 & +7 & -14 & $\begin{array}{l}+7 \\
\end{array}$ & $\overline{-1}$ & \\
\hline (72) & +1 & +8 & -2 & +3 & -13 & +11 & +5 & $\begin{array}{l}+5 \\
\end{array}$ & -10 & +2 & -5 & +5 & -1 & & \\
\hline (63) & $\begin{array}{l}-9 \\
\end{array}$ & +12 & -3 & -6 & +18 & -3 & -3 & -9 & +3 & $\ldots$ & +3 & -1 & & & \\
\hline (54) & +3 & -4 & $\ldots$ & +3 & -7 & -2 & +1 & +4 & $\ldots$ & $\ldots$ & -1 & & & & \\
\hline$\left(71^{2}\right)$ & +5 & -5 & +1 & -3 & +12 & -6 & +2 & $\begin{array}{l}-9 \\
-\quad 9\end{array}$ & +6 & -1 & & & & & \\
\hline (621) & +12 & -14 & +3 & +3 & -7 & $\begin{array}{l}+1 \\
\end{array}$ & -2 & $\begin{array}{l}+4 \\
+4\end{array}$ & -1 & & & & & & \\
\hline (531) & -2 & $\begin{array}{l}+1 \\
\end{array}$ & $\ldots$ & +3 & -4 & +2 & +2 & -1 & & & & & & & \\
\hline$\left(4^{2} 1\right)$ & +1 & $\ldots$ & $\ldots$ & -3 & $\begin{array}{l}+3 \\
\end{array}$ & $\ldots$ & -1 & & & & & & & & \\
\hline$\left(52^{2}\right)$ & -6 & $\begin{array}{l}+\quad 2 \\
+\end{array}$ & $\ldots$ & -3 & $\begin{array}{l}+3 \\
\end{array}$ & -1 & & & & & & & & & \\
\hline (432) & $\begin{array}{l}+\quad 2 \\
\end{array}$ & $\bar{\ldots}$ & $\ldots$ & +3 & -1 & & & & & & & & & & \\
\hline$\left(3^{3}\right)$ & $\ldots$ & $\ldots$ & $\ldots$ & -1 & & & & & & & & & & & \\
\hline$\left(61^{3}\right)$ & -5 & $\begin{array}{l}+5 \\
\end{array}$ & -1 & & & & & & & & & & & & \\
\hline$\left(521^{2}\right)$ & +3 & -1 & & & & & & & & & & & & & \\
\hline$\left(431^{2}\right)$ & -1 & & & & & & & & & & & & & & \\
\hline & & & & & & & & & & & & & & & \\
\hline & & & & & & & & & & & & & & & \\
\hline & & & & & & & & & & & & & & & \\
\hline & & & & & & & & & & & & & & & \\
\hline & & & & & & & & & & & & & & & \\
\hline & & & & & & & & & & & & & & & \\
\hline & & & & & & & & & & & & & & & \\
\hline & & & & & & & & & & & & & & & \\
\hline & & & & & & & & $=$ & & & & & & & \\
\hline & & & & & & & & & & & & & & & \\
\hline & & & & & & & & & & & & & & & \\
\hline & & & & & & & & & & & & & & & \\
\hline & & & & & & & & & & & & & & & \\
\hline & & & & & & & & & & & & & & & \\
\hline & & & & & & & & & & & & & & & \\
\hline
\end{tabular}


$X(a)$. Runs on to p. 436 .

\begin{tabular}{|c|c|c|c|c|c|c|c|c|c|c|c|c|c|}
\hline ॥ & $\begin{array}{c}10 \\
k\end{array}$ & $\begin{array}{l}19 \\
b j\end{array}$ & $\begin{array}{l}28 \\
c i\end{array}$ & $\begin{array}{l}1^{2} 8 \\
b^{2} i\end{array}$ & $\begin{array}{l}37 \\
d h\end{array}$ & $\begin{array}{l}127 \\
b c h\end{array}$ & $\begin{array}{l}1^{37} 7 \\
b^{3} h\end{array}$ & $\begin{array}{l}46 \\
e g\end{array}$ & $\begin{array}{l}136 \\
b d g\end{array}$ & $\begin{array}{l}2^{2} 6 \\
c^{2} g\end{array}$ & $\begin{array}{l}1^{2} 26 \\
b^{2} \mathrm{cg}\end{array}$ & $\begin{array}{l}1^{4} 6 \\
b^{4} g\end{array}$ & $\begin{array}{l}5^{2} \\
f^{2}\end{array}$ \\
\hline (10) & & & & & & & & & & & & & \\
\hline (91) & 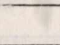 & & & & & & & & & & & & t \\
\hline (82) & & & & & & & & & & & & & 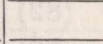 \\
\hline (73) & & & & & & & & & & & & & $\square$ \\
\hline (64) & & & & & & & & & & & & & + \\
\hline$\left(5^{2}\right)$ & & & & & & & & & & & & & 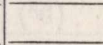 \\
\hline$\left(81^{2}\right)$ & & & & & & & & & & & & & \\
\hline (721) & & & & & & & & & & & & & \\
\hline (631) & & & & & & & & & & & & & T \\
\hline (541) & & & & & & $\pi$ & & & & & & & $\square$ \\
\hline$\left(62^{2}\right)$ & & & & & & & & & & & & & \\
\hline (532) & & & & & & & & & & & & & \\
\hline$\left(4^{2} 2\right)$ & & & 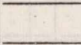 & & & & & & & & & & 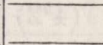 \\
\hline$\left(43^{2}\right)$ & & & & & & & & & & & & & T \\
\hline$\left(71^{3}\right)$ & & & & & & & & & & & & & 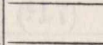 \\
\hline$\left(621^{2}\right)$ & & & & & & & & & & & & & \\
\hline$\left(531^{2}\right)$ & & & 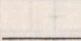 & & & & & & & & & & 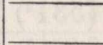 \\
\hline$\left(4^{2} 1^{2}\right)$ & & & & & & & & & & & & & \\
\hline$\left(52^{2} 1\right)$ & & & & & & & & & & & & & \\
\hline (4321) & & & E & & & & & & & & E. & & \\
\hline$\left(3^{3} 1\right)$ & & & Z & & & & & & & & & & \\
\hline$\left(42^{3}\right)$ & & & 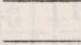 & & & & & & & & & & \\
\hline$\left(3^{2} 2^{2}\right)$ & & & E & & & & & & & & & & \\
\hline$\left(61^{4}\right)$ & & & 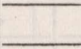 & & & & & & & & & & \\
\hline$\left(521^{3}\right)$ & & & 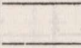 & & & & & & & & & & \\
\hline$\left(431^{3}\right)$ & & & & & & & & & & & & & \\
\hline$\left(42^{2} 1^{2}\right)$ & & & 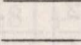 & & & & & & & & & & \\
\hline$\left(3^{2} 21^{2}\right)$ & & & 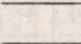 & & & & & & & & & & \\
\hline$\left(32^{3} 1\right)$ & & & & & & & & & & 7 & & & \\
\hline$\left(2^{5}\right)$ & & & 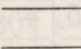 & & & & & & & & & & +1 \\
\hline$\left(51^{5}\right)$ & & & 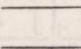 & & & & & & & & & + & $\ldots$ \\
\hline$\left(421^{4}\right)$ & & & & & & & & & & & $+\quad 1$ & + & $\ldots$ \\
\hline$\left(3^{2} 1^{4}\right)$ & & & & & & & & & & + & + & + & $\ldots$ \\
\hline$\left(32^{2} 1^{3}\right)$ & & & & & +1 & & & & +1 & + & $\begin{array}{l}+5 \\
\end{array}$ & 12 & $\ldots$ \\
\hline$\left(2^{4} 1^{2}\right)$ & & & & & & 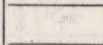 & & +1 & +4 & + & +12 & +24 & +2 \\
\hline$\left(41^{6}\right)$ & 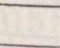 & & 5 & & & & +1 & $\ldots$ & $\ldots$ & $\ldots$ & +6 & $+\quad 25$ & $\ldots$ \\
\hline$\left(321^{5}\right)$ & & & & & & +1 & +3 & $\ldots$ & +5 & +11 & + & 70 & $\ldots$ \\
\hline$\left(2^{3} 1^{4}\right)$ & & & & & +1 & $\begin{array}{r}+3 \\
\end{array}$ & +6 & +4 & +19 & + & $\begin{array}{l}+63 \\
\end{array}$ & +132 & +6 \\
\hline$\left(31^{7}\right)$ & 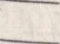 & & & +1 & $\ldots$ & $\begin{array}{l}+7 \\
+\quad 7\end{array}$ & +22 & $\ldots$ & +21 & +42 & +112 & +280 & $\ldots$ \\
\hline$\left(2^{2} 1^{6}\right)$ & & & +1 & +2 & +6 & +20 & +42 & +15 & +72 & +115 & +242 & +510 & +20 \\
\hline$\left(21^{8}\right)$ & & +1 & +8 & +17 & +28 & +92 & +192 & +56 & +252 & +392 & +812 & +1680 & +70 \\
\hline$\left(1^{10}\right)$ & +1 & +10 & +45 & +90 & +120 & +360 & +720 & +210 & $|\overline{+840}|$ & +1260 & $\mid+2520$ & +5040 & +252 \\
\hline
\end{tabular}




\begin{tabular}{|c|c|c|c|c|c|c|c|c|c|c|}
\hline$\|$ & $\begin{array}{l}145 \\
\text { bef }\end{array}$ & $\begin{array}{l}235 \\
c d f\end{array}$ & $\begin{array}{l}1^{2} 35 \\
b^{2} d f^{4}\end{array}$ & $\begin{array}{l}12^{2} 5 \\
b c^{2} f\end{array}$ & $\begin{array}{l}1325 \\
b^{3} c f\end{array}$ & $\begin{array}{l}1^{5} 5 \\
b^{5} f\end{array}$ & $\begin{array}{l}24^{2} \\
c e^{2} \\
\end{array}$ & $\begin{array}{l}1^{2} 4^{2} \\
b^{2} e^{2} \\
\end{array}$ & $\begin{array}{l}3^{2} 4 \\
d^{2} e \\
\end{array}$ & $\begin{array}{l}1234 \\
\text { bcde }\end{array}$ \\
\hline (10) & & & & & & & & & & \\
\hline (91) & & & & & & & & & & \\
\hline (82) & & & & & & & & & & \\
\hline (73) & & & & & & & & & & \\
\hline (64) & & & & & & & & & & \\
\hline$\left(5^{2}\right)$ & & & & & & & & & & \\
\hline$\left(81^{2}\right)$ & & & & & $\beta$ & & & & & \\
\hline (721) & & & & & & & & & & \\
\hline (631) & & & & & & & & & & \\
\hline (541) & & & & & & & & & & \\
\hline$\left(62^{2}\right)$ & & & & & & & & & & \\
\hline (532) & & & & & & & & & & \\
\hline$\left(4^{2} 2\right)$ & & & & & & & & & & \\
\hline$\left(43^{2}\right)$ & & & & & & & & & & \\
\hline$\left(71^{3}\right)$ & & & & & & & & & & \\
\hline$\left(621^{2}\right)$ & & a & & & & & & & & \\
\hline$\left(531^{2}\right)$ & & & & & & & & & & \\
\hline$\left(4^{2} 1^{2}\right)$ & & & & & & & & & & \\
\hline$\left(52^{2} 1\right)$ & & & & & & & & & & \\
\hline (4321) & & & & & & & & & & + \\
\hline$\left(3^{3} 1\right)$ & & & & & & & & & + & + \\
\hline$\left(42^{3}\right)$ & & & & & & & & + & $\ldots$ & + \\
\hline$\left(3^{2} 2^{2}\right)$ & & & & & & & + & + & +2 & + \\
\hline$\left(61^{4}\right)$ & & & & & & + & $\ldots$ & $\ldots$ & $\ldots$ & $\ldots$ \\
\hline$\left(521^{3}\right)$ & & & & & 1 & + & $\ldots$ & $\ldots$ & $\ldots$ & $\ldots$ \\
\hline$\left(431^{3}\right)$ & & & & + & 3 & + & $\ldots$ & $\ldots$ & $\ldots$ & + \\
\hline$\left(42^{2} 1^{2}\right)$ & & & + & 2 & 7 & 20 & $\ldots$ & $\begin{array}{l}+\quad 2 \\
\end{array}$ & $\ldots$ & + \\
\hline$\left(3^{2} 21^{2}\right)$ & & 1 & + & 5 & 12 & 30 & $+\quad 2$ & + & +5 & 21 \\
\hline$\left(32^{3} 1\right)$ & +1 & $\begin{array}{l}+\quad 3 \\
\end{array}$ & + & 12 & 27 & 60 & + & $\begin{array}{l}+\quad 16 \\
\end{array}$ & $\begin{array}{l}+\quad 12 \\
\end{array}$ & 49 \\
\hline$\left(2^{5}\right)$ & +5 & $+\quad 10$ & $+\quad 20$ & $\begin{array}{l}+\quad 30 \\
\end{array}$ & 60 & 120 & $\begin{array}{l}+\quad 20 \\
\end{array}$ & $\begin{array}{l}+45 \\
\end{array}$ & $\begin{array}{l}+30 \\
\end{array}$ & $+\quad 110$ \\
\hline$\left(51^{5}\right)$ & $\ldots$ & $\ldots$ & $\ldots$ & $\ldots$ & 5 & 26 & $\ldots$ & $\ldots$ & $\ldots$ & $\ldots$ \\
\hline$\left(421^{4}\right)$ & $\ldots$ & $\ldots$ & +4 & $\begin{array}{l}+\quad 9 \\
\end{array}$ & 32 & 95 & $\ldots$ & $\begin{array}{l}+\quad 6 \\
\end{array}$ & $\ldots$ & \\
\hline$\left(3^{2} 1^{4}\right)$ & $\ldots$ & & + & 22 & 54 & 140 & $\begin{array}{l}+6 \\
\end{array}$ & $\begin{array}{r}+\quad 12 \\
\end{array}$ & $\begin{array}{l}+\quad 12 \\
\end{array}$ & 56 \\
\hline$\left(32^{2} 1^{3}\right)$ & $+\quad 3$ & $+\quad 11$ & $\begin{array}{l}+\quad 26 \\
\end{array}$ & $\begin{array}{l}48 \\
\end{array}$ & 112 & 260 & $+\quad 18$ & $\begin{array}{r}+\quad 42 \\
\end{array}$ & $+\quad 31$ & 128 \\
\hline$\left(2^{4} 1^{2}\right)$ & $+\quad 14$ & $\begin{array}{l}+32 \\
\end{array}$ & $\begin{array}{l}+68 \\
\end{array}$ & +108 & 228 & 480 & +53 & +114 & $\begin{array}{l}+\quad 80 \\
\end{array}$ & 284 \\
\hline$\left(41^{6}\right)$ & $\ldots$ & $\ldots$ & $+\quad 15$ & $+\quad 30$ & 111 & 330 & $\ldots$ & $\begin{array}{l}+\quad 20 \\
\end{array}$ & $\ldots$ & \\
\hline$\left(321^{5}\right)$ & $+\quad 10$ & $\begin{array}{l}+35 \\
\end{array}$ & +85 & +156 & 368 & 860 & +50 & +120 & +80 & 335 \\
\hline$\left(2^{3} 1^{4}\right)$ & $\begin{array}{l}+42 \\
+\quad \\
\end{array}$ & $\begin{array}{l}+99 \\
\end{array}$ & +210 & +339 & 720 & 1530 & +144 & $\begin{array}{l}+306 \\
\end{array}$ & +213 & 735 \\
\hline$\left(31^{7}\right)$ & $\begin{array}{l}+\quad 35 \\
\end{array}$ & +105 & $\begin{array}{l}+266 \\
\end{array}$ & +462 & +1092 & 2520 & +140 & $+\quad 350$ & +210 & $+\quad 875$ \\
\hline$\left(2^{2} 1^{6}\right)$ & +130 & $\begin{array}{r}+\quad 296 \\
\end{array}$ & $\begin{array}{l}+622 \\
\end{array}$ & $\begin{array}{r}+990 \\
\end{array}$ & +2082 & +4380 & +400 & +840 & +570 & +1900 \\
\hline$\left(21^{8}\right)$ & +406 & $\begin{array}{l}+868 \\
\end{array}$ & +1792 & +2772 & +5712 & +11760 & +1120 & +2310 & +1540 & +4900 \\
\hline$\left(1^{10}\right)$ & +1260 & +2520 & +5040 & +7560 & +15120 & $\overline{+30240}$ & +3150 & +6300 & +4200 & +12600 \\
\hline
\end{tabular}




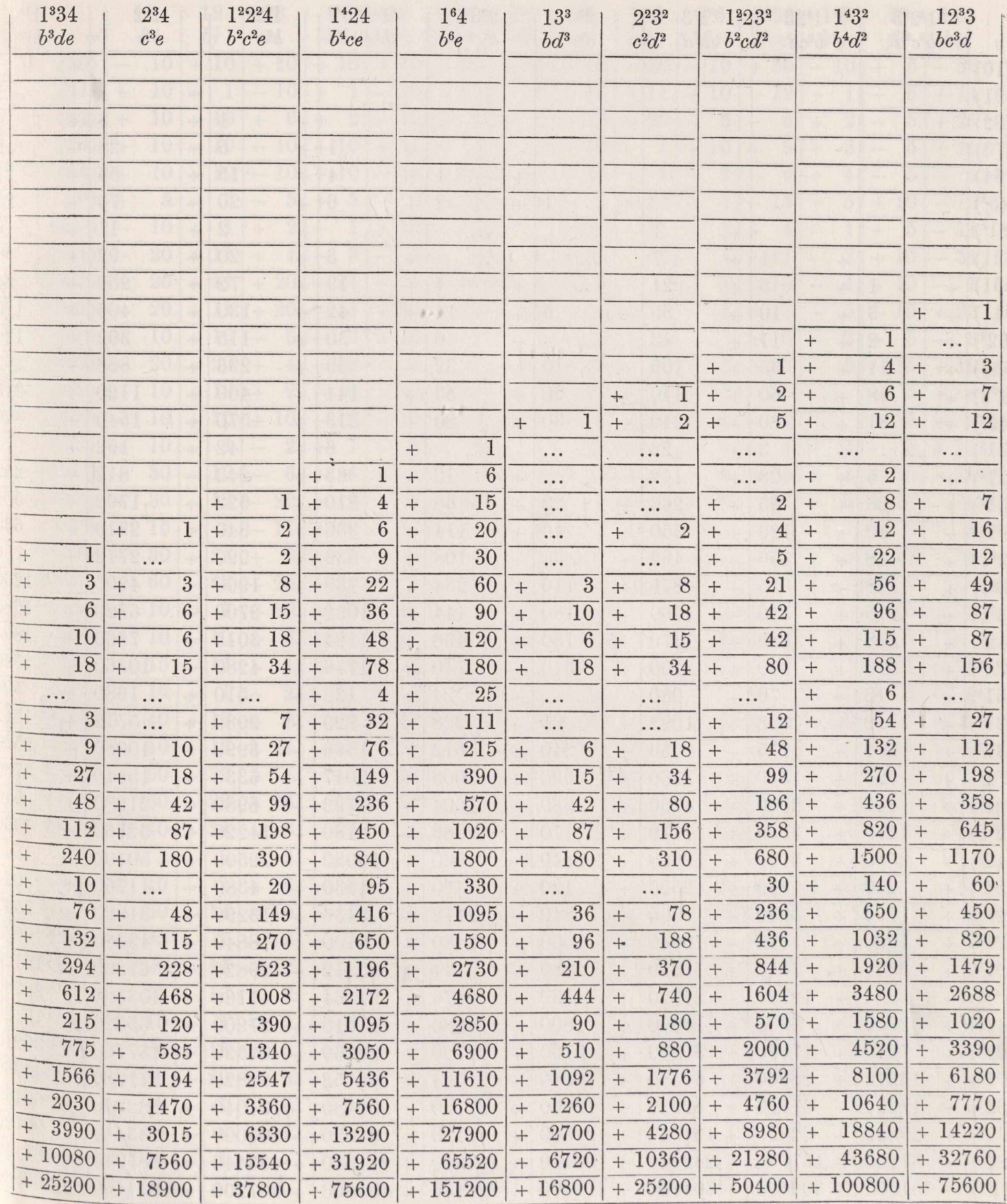




\begin{tabular}{|c|c|c|c|c|c|c|c|c|c|c|c|c|c|c|c|c|c|c|}
\hline II & & $\begin{array}{l}1^{3} 2^{23} \\
b^{3} c^{2} d\end{array}$ & & $\begin{array}{l}1^{5} 23 \\
b^{5} c d\end{array}$ & & $\begin{array}{l}1^{7} 3 \\
b^{7} d\end{array}$ & & $\begin{array}{l}2^{5} \\
c^{5}\end{array}$ & & $\begin{array}{l}2^{2} 3^{2} \\
b^{2} c^{4}\end{array}$ & & $\begin{array}{l}1^{4} 2^{3} \\
b^{4} c^{3}\end{array}$ & & $\begin{array}{l}1^{6} 2^{2} \\
b^{6} c^{2}\end{array}$ & & $\begin{array}{l}1^{8} 2 \\
b^{8} c\end{array}$ & & $\begin{array}{l}1^{10} \\
b^{10}\end{array}$ \\
\hline (10) & & & & & & & & & & & & & & & & & + & 1 \\
\hline (91) & & & & & & & & & & & & & & & + & 1 & + & 10 \\
\hline (82) & & & & & & & & $E$ & & & & & - & 1 & + & 8 & + & 45 \\
\hline (73) & & & & & & & & & & & & 1 & & 6 & + & 28 & + & 120 \\
\hline (64) & & & & & & & & & + & 1 & - & 4 & - & 15 & + & 56 & + & 210 \\
\hline$\left(5^{2}\right)$ & & & & & & & + & 1 & $t$ & 2 & & 6 & & 20 & + & 70 & + & 252 \\
\hline$\left(81^{2}\right)$ & & & & & + & 1 & & $\ldots$ & & $\ldots$ & & $\ldots$ & - & 2 & + & 17 & + & 90 \\
\hline (721) & & & + & 1 & + & 7 & & $\ldots$ & & $\ldots$ & $t$ & 3 & - & 20 & + & 92 & + & 360 \\
\hline (631) & + & 1 & + & 5 & + & 21 & & $\ldots$ & + & 4 & + & $\overline{19}$ & - & 72 & + & 252 & + & 840 \\
\hline (541) & + & 3 & t & 10 & + & 35 & + & 5 & $t$ & 14 & - & 42 & . & 130 & + & 406 & & 1260 \\
\hline$\left(62^{2}\right)$ & + & 2 & + & 11 & + & 42 & & $\ldots$ & + & 6 & + & 30 & & 115 & + & 392 & & 1260 \\
\hline$(532)$ & + & 11 & + & 35 & + & 105 & + & 10 & - & 32 & & 99 & . & 296 & + & 868 & $T$ & 2520 \\
\hline$\left(4^{2} 2\right)$ & + & 18 & + & 50 & + & 140 & + & 20 & t & 53 & & 144 & . & 400 & + & 1120 & + & 3150 \\
\hline$\left(43^{2}\right)$ & + & 31 & + & 80 & + & 210 & + & 30 & + & 80 & -1 & 213 & & 570 & + & 1540 & & 4200 \\
\hline$\left(71^{3}\right)$ & & $\ldots$ & + & 3 & + & 22 & & $\ldots$ & & $\ldots$ & + & 6 & & 42 & + & 192 & $T$ & 720 \\
\hline$\left(621^{2}\right)$ & + & 5 & + & 28 & + & 112 & & $\ldots$ & + & 12 & + & 63 & & 242 & + & 812 & + & 2520 \\
\hline$\left(531^{2}\right)$ & + & 26 & + & 85 & + & 266 & + & 20 & $t$ & 68 & - & 210 & & 622 & 4 & 1792 & + & 5040 \\
\hline$\left(4^{2} 1^{2}\right)$ & + & 42 & + & 120 & + & 350 & + & 45 & + & 114 & 7 & 306 & & 840 & + & 2310 & & 6300 \\
\hline$\left(52^{2} 1\right)$ & + & 48 & + & 156 & + & 462 & + & 30 & + & 108 & + & 339 & . & 990 & + & 2772 & + & 7560 \\
\hline 4321) & + & 128 & + & 335 & + & 875 & + & 110 & - & 284 & & 735 & & 1900 & + & 4900 & & 12600 \\
\hline$\left(3^{3} 1\right)$ & + & 210 & $t$ & 510 & + & 1260 & + & 180 & + & 444 & & 1092 & & 2700 & + & 6720 & & 16800 \\
\hline$\left(42^{3}\right)$ & + & 228 & + & 585 & + & 1470 & + & 180 & 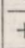 & 468 & - & 1194 & & 3015 & + & 7560 & + & 18900 \\
\hline$\left(3^{2} 2^{2}\right)$ & + & 370 & + & 880 & + & 2100 & + & 310 & - & 740 & & 1776 & & 4280 & + & 10360 & & 25200 \\
\hline$\left(61^{4}\right)$ & + & 12 & + & 70 & + & 280 & & $\ldots$ & - & 24 & - & 132 & & 510 & + & 1680 & $t$ & 5040 \\
\hline$\left(521^{3}\right)$ & + & 112 & + & 368 & + & 1092 & + & 60 & - & 228 & + & 720 & . & 2082 & + & 5712 & + & 15120 \\
\hline$\left(431^{3}\right)$ & + & 294 & $t$ & 775 & $t$ & 2030 & + & 240 & - & 612 & - & 1566 & & 3990 & + & 10080 & & 25200 \\
\hline $\left.42^{2} 1^{2}\right)$ & + & 523 & + & 1340 & + & 3360 & + & 390 & - & 1008 & - & 2547 & & 6330 & + & 15540 & + & 37800 \\
\hline$\left(3^{2} 21^{2}\right)$ & + & 844 & $t$ & 2000 & + & 4760 & + & 680 & $t$ & 1604 & - & 3792 & & 8980 & + & 21280 & - & 50400 \\
\hline$\left(32^{3} 1\right)$ & + & 1479 & + & 3390 & $t$ & 7770 & + & 1170 & - & 2688 & + & 6180 & & -14220 & + & 32760 & & 75600 \\
\hline$\left(2^{5}\right)$ & + & 2580 & + & 5700 & & 12600 & + & 2040 & - & 4530 & - & 10080 & & -22500 & + & 50400 & $t$ & 113400 \\
\hline$\left(51^{5}\right)$ & + & 260 & + & 860 & + & 2520 & + & 120 & + & 480 & - & 1530 & & -4380 & + & 11760 & $t$ & 30240 \\
\hline$\left(421^{4}\right)$ & & 1196 & $t$ & 3050 & $t$ & 7560 & + & 840 & - & 2172 & $t$ & 5436 & & -13290 & + & 31920 & & 75600 \\
\hline$\left(3^{2} 1^{4}\right)$ & + & 1920 & t & 4520 & & 10640 & + & 1500 & + & 3480 & - & 8100 & & -18840 & + & 43680 & $t$ & 100800 \\
\hline $\left.32^{2} 1^{3}\right)$ & + & 3358 & + & 7610 & + & 17220 & + & 2580 & $f$ & 5844 & $t$ & 13212 & & -29820 & + & 67200 & & 151200 \\
\hline$\left(2^{4} 1^{2}\right)$ & + & 5844 & & 12720 & + & 27720 & + & 4530 & $t$ & 9876 & & 21564 & & -47160 & + & 103320 & & 226800 \\
\hline$\left(41^{6}\right)$ & + & 2730 & & 6900 & + & 16800 & + & 1800 & $t$ & 4680 & & 11610 & & -27900 & + & 65520 & & 151200 \\
\hline$\left(321^{5}\right)$ & + & 7610 & & 17000 & - & 37800 & + & 5700 & $t$ & 12720 & & 28260 & & -62520 & + & 137760 & & 302400 \\
\hline$\left(2^{3} 1^{4}\right)$ & & 13212 & & 28260 & - & 60480 & + & 10080 & $f$ & 21564 & & 46152 & & -98820 & + & 211680 & & 453600 \\
\hline$\left(31^{7}\right)$ & & 17220 & & 37800 & & 82320 & + & 12600 & -1 & 27720 & & 60480 & & -131040 & + & 282240 & & 604800 \\
\hline$\left(2^{2} 1^{6}\right)$ & + & 29820 & & 62520 & & 131040 & + & 22500 & & 47160 & & 98820 & & -207000 & + & 433440 & & 907200 \\
\hline$\left(21^{8}\right)$ & & 67200 & & 137760 & & 282240 & + & 50400 & & 103320 & & 211680 & & -433440 & & 887040 & & 1814400 \\
\hline$\left(1^{10}\right)$ & + & 151200 & & 302400 & & 604800 & $\overline{+1}$ & 113400 & & 226800 & & 453600 & & -907200 & & 1814400 & & 3628800 \\
\hline
\end{tabular}




\section{X (b). Runs on to p. 439.}

\begin{tabular}{|c|c|c|c|c|c|c|c|c|c|c|c|c|c|c|}
\hline & $\begin{array}{c}10 \\
k\end{array}$ & $\begin{array}{l}19 \\
b j\end{array}$ & $\begin{array}{l}28 \\
c i\end{array}$ & $\begin{array}{l}1^{2} 8 \\
b^{2} i\end{array}$ & $\begin{array}{l}37 \\
d h\end{array}$ & bch & $b^{3} h$ & $\begin{array}{l}46 \\
e g\end{array}$ & $b d g$ & $\begin{array}{l}2^{2} 6 \\
c^{2} g\end{array}$ & $b^{2} c g$ & $b^{4} g$ & $f^{2}$ & bef \\
\hline (10) & -10 & +10 & +10 & -10 & +10 & -20 & +10 & +10 & -20 & -10 & +30 & -10 & $\begin{array}{l}+5 \\
\end{array}$ & -20 \\
\hline (91) & +10 & -1 & -10 & +1 & -10 & +11 & -1 & -10 & $\overline{+11}$ & +10 & -12 & +1 & -5 & +11 \\
\hline (82) & 10 & -10 & $\begin{array}{l}+6 \\
\end{array}$ & +2 & -10 & +4 & -2 & -10 & +20 & -6 & -6 & $\begin{array}{l}+\quad 2 \\
\end{array}$ & $=$ & +20 \\
\hline (73) & 10 & -10 & -10 & +10 & +11 & -1 & -3 & $\overline{-10}$ & -1 & +10 & -9 & +3 & -5 & +20 \\
\hline (64) & 10 & -10 & -10 & +10 & -10 & +20 & -10 & +14 & -4 & -2 & -6 & +4 & -5 & -4 \\
\hline$\left(5^{2}\right)$ & 5 & -5 & $\overline{-5}$ & +5 & -5 & +10 & -5 & -5 & +10 & +5 & -15 & +5 & $\overline{+10}$ & -15 \\
\hline$\left(81^{2}\right)$ & -10 & +1 & +2 & -1 & +10 & -3 & +1 & +10 & -11 & -2 & $\begin{array}{r}+\quad 4 \\
\end{array}$ & -1 & +5 & -11 \\
\hline 1) & 20 & +11 & +4 & -3 & -1 & -8 & & +20 & -10 & -4 & +11 & -3 & +10 & -31 \\
\hline & 20 & +11 & +20 & -11 & -1 & -10 & +4 & -4 & $\overline{-4}$ & -8 & +15 & -4 & +10 & -7 \\
\hline & 20 & +11 & +20 & -11 & +20 & -31 & +11 & - & -7 & -8 & +18 & -5 & -15 & +23 \\
\hline $\left.2^{2}\right)$ & 10 & +10 & -6 & -2 & +10 & -4 & & -2 & -8 & $\ldots$ & $\begin{array}{l}+6 \\
+\quad 6\end{array}$ & -2 & +5 & -8 \\
\hline & 20 & +20 & +4 & $\overline{-12}$ & -1 & -3 & +5 & +20 & -19 & -4 & +15 & -5 & -15 & +10 \\
\hline 2) & 10 & +10 & $\begin{array}{l}+\quad 2 \\
\end{array}$ & -6 & +10 & -12 & + & -14 & +4 & +10 & -6 & $\ldots$ & $\begin{array}{l}+5 \\
\end{array}$ & +4 \\
\hline $\left.3^{2}\right)$ & -10 & +10 & +10 & $\overline{-10}$ & -11 & +1 & +3 & -2 & $\overline{+13}$ & -4 & -3 & $\ldots$ & + & -8 \\
\hline & 10 & -1 & -2 & +1 & -3 & +3 & -1 & -10 & +4 & +2 & -4 & +1 & -5 & +11 \\
\hline 62 & +30 & -12 & $\overline{-6}$ & +4 & $\overline{-9}$ & +11 & $\overline{-4}$ & -6 & +15 & +6 & -15 & +4 & -15 & +18 \\
\hline & +30 & -12 & -22 & +12 & -9 & +13 & -5 & $\overline{-6}$ & +15 & +10 & -19 & +5 & +.10 & -12 \\
\hline & +15 & -6 & -11 & $\begin{array}{r}+6 \\
\end{array}$ & -15 & +17 & -6 & +9 & -3 & -1 & +1 & $\bar{\ldots}$ & +5 & -8 \\
\hline $\left.52^{2} 1\right)$ & 30 & -21 & +2 & +5 & -9 & +12 & -5 & -18 & +18 & +4 & -17 & +5 & +10 & -1 \\
\hline & +60 & -42 & $\overline{-28}$ & +26 & +3 & +21 & -12 & +12 & -15 & -8 & +7 & $\ldots$ & -5 & $\ldots$ \\
\hline$\left.{ }^{3} 1\right)$ & 10 & -7 & $\overline{-10}$ & +7 & +11 & -4 & $\ldots$ & +2 & -7 & +4 & $\ldots$ & $\ldots$ & - & +5 \\
\hline (4 & +10 & -10 & $\begin{array}{l}+6 \\
\end{array}$ & +2 & -10 & +4 & -2 & +10 & $\ldots$ & -4 & $\begin{array}{l}+2 \\
+\end{array}$ & $\ldots$ & - & $\ldots$ \\
\hline $\left.2^{2}\right)$ & +15 & -15 & +1 & +7 & +6 & -7 & $\ldots$ & -9 & $\begin{array}{l}+3 \\
\end{array}$ & +2 & $\ldots$ & $\ldots$ & + & -1 \\
\hline $\left.1^{4}\right)$ & 10 & +1 & 2 & -1 & +3 & -3 & +1 & +4 & -4 & -2 & +4 & -1 & + & - \\
\hline $\left.521^{3}\right)$ & -40 & +13 & +8 & -5 & +12 & -14 & +5 & +16 & -19 & -8 & +19 & -5 & - & + \\
\hline $\left.431^{3}\right)$ & -40 & $\overline{+13}$ & +24 & -13 & +12 & -16 & $\overline{+6}$ & -8 & +5 & $\bar{\ldots}$ & -1 & $\ldots$ & - & + \\
\hline & -60 & +33 & +4 & -9 & +18 & -23 & $\begin{array}{l}+9 \\
\end{array}$ & -12 & + & +8 & -4 & $\ldots$ & + & - \\
\hline$\left.{ }^{2} 21^{2}\right)$ & -60 & +33 & +28 & -21 & -24 & $\begin{array}{l}+9 \\
\end{array}$ & $\begin{array}{l}\ldots \\
\end{array}$ & $\ldots$ & +6 & -4 & $\ldots$ & $\ldots$ & + & - \\
\hline 32 & -40 & +31 & -8 & -7 & -2 & +5 & $\ldots$ & +8 & -3 & $\bar{\ldots}$ & $\ldots$ & $\ldots$ & - & + \\
\hline$\left(2^{5}\right)$ & $\begin{array}{l}-\quad 2 \\
\end{array}$ & $\begin{array}{l}+\quad 2 \\
\end{array}$ & -2 & $\ldots$ & +2 & $\ldots$ & $\ldots$ & -2 & $\ldots$ & $\ldots$ & $\ldots$ & $\ldots$ & +1 & \\
\hline $\left.1^{5}\right)$ & +10 & -1 & -2 & +1 & -3 & +3 & -1 & - & +4 & +2 & -4 & +1 & & \\
\hline 42 & +50 & $\overline{-14}$ & -10 & +6 & -15 & +17 & -6 & +4 & - & -2 & +1 & & & \\
\hline $\left.1^{4}\right)$ & +25 & -7 & -13 & $\begin{array}{l}+7 \\
+\end{array}$ & +3 & -1 & $\ldots$ & + & - & +1 & & & & \\
\hline & +100 & -46 & -12 & +14 & +12 & -5 & $\ldots$ & -4 & +1 & & & & & \\
\hline $\left.1^{2}\right)$ & +25 & -16 & +9 & $\ldots$ & -4 & $\ldots$ & $\ldots$ & +1 & & & & & & \\
\hline (4 & -10 & +1 & +2 & -1 & $\begin{array}{l}+3 \\
\end{array}$ & -3 & +1 & & & & & & & \\
\hline & -60 & +15 & +12 & -7 & -3 & +1 & & & & & & & & \\
\hline & 50 & +20 & -6 & $\ldots$ & +1 & & & & & & & & & \\
\hline & +10 & -1 & -2 & +1 & & & & & & & & & & \\
\hline & +35 & -8 & +1 & & & & & & & & & & & \\
\hline & -10 & +1 & & & & & & & & & & & & \\
\hline & +1 & & & & & & & & & & & & & \\
\hline
\end{tabular}




\begin{tabular}{|c|c|c|c|c|c|c|c|c|c|c|c|c|c|c|}
\hline$=$ & $\begin{array}{l}235 \\
c d f\end{array}$ & $\begin{array}{l}1^{2} 35 \\
b^{2} d f\end{array}$ & $\begin{array}{l}12^{2} 5 \\
b c^{2} f\end{array}$ & $\begin{array}{l}1^{3} 25 \\
b^{3} c f\end{array}$ & $\begin{array}{l}1^{5} 5 \\
b^{5} f\end{array}$ & $\begin{array}{l}24^{2} \\
c e^{2}\end{array}$ & $\begin{array}{l}1^{2} 4^{2} \\
b^{2} e^{2}\end{array}$ & $\begin{array}{l}3^{2} 4 \\
d^{2} e\end{array}$ & $\begin{array}{l}1234 \\
\text { bcde }\end{array}$ & $\begin{array}{l}1^{3} 34 \\
b^{3} d e\end{array}$ & $\begin{array}{l}2^{3} 4 \\
c^{3} e\end{array}$ & $\begin{array}{l}1^{2} 2^{2} 4 \\
b^{2} c^{2} e\end{array}$ & $\begin{array}{l}1^{4} 24 \\
b^{4} c e\end{array}$ & $\begin{array}{l}1^{6} 4 \\
b^{6} e\end{array}$ \\
\hline (10) & -20 & +30 & +30 & -40 & +10 & -10 & +15 & -10 & +60 & $\overline{-40}$ & $\overline{+10}$ & -60 & +50 & -10 \\
\hline (91) & +20 & -12 & $\overline{-21}$ & +13 & -1 & +10 & -6 & +10 & -42 & +13 & -10 & +33 & -14 & +1 \\
\hline (82) & +4 & -22 & +2 & +8 & -2 & +2 & -11 & +10 & -28 & +24 & +6 & +4 & -10 & + \\
\hline (73) & -1 & -9 & -9 & +12 & -3 & +10 & -15 & -11 & +3 & +12 & $\overline{-10}$ & +18 & -15 & +3 \\
\hline (64) & +20 & -6 & -18 & +16 & -4 & -14 & $\begin{array}{l}+\quad 9 \\
\end{array}$ & -2 & +12 & -8 & $\overline{+10}$ & -12 & +4 & $\ldots$ \\
\hline$\left(5^{2}\right)$ & -15 & +10 & +10 & -5 & $\ldots$ & $+\tilde{5}$ & +5 & +5 & -5 & -5 & -5 & +5 & $\ldots$ & $\ldots$ \\
\hline$\left(81^{2}\right)$ & -12 & +12 & +5 & -5 & +1 & -6 & +6 & -10 & +26 & $\overline{-13}$ & +2 & -9 & +6 & -1 \\
\hline$(721)$ & -3 & +13 & +12 & -14 & +3 & -12 & +17 & +1 & +21 & -16 & $\overline{+4}$ & -23 & +17 & -3 \\
\hline (631) & -19 & +15 & +18 & -19 & +4 & +4 & -3 & +13 & -15 & +5 & $\ldots$ & +3 & -1 & $\ldots$ \\
\hline (541) & +10 & -12 & -1 & +1 & $\ldots$ & +4 & -8 & -8 & $\ldots$ & +5 & $\ldots$ & -1 & $\ldots$ & $\ldots$ \\
\hline$\left(62^{2}\right)$ & -4 & +10 & +4 & -8 & +2 & +10 & -1 & - & -8 & $\ldots$ & $\overline{-4}$ & + & -2 & $\ldots$ \\
\hline (532) & +17 & -4 & -13 & +5 & $\ldots$ & -12 & +1 & & +5 & -1 & +4 & -2 & $\ldots$ & $\ldots$ \\
\hline$\left(4^{2} 2\right)$ & -12 & +2 & +2 & $\ldots$ & $\ldots$ & +2 & -3 & +2 & +4 & $\ldots$ & -2 & $\ldots$ & $\ldots$ & $\ldots$ \\
\hline$\left(43^{2}\right)$ & +1 & -3 & +3 & $\ldots$ & $\ldots$ & & +3 & - & $\overline{-3}$ & $\ldots$ & $\ldots$ & $\ldots$ & $\ldots$ & $\ldots$ \\
\hline$\left(71^{3}\right)$ & +5 & -5 & -5 & +5 & -1 & & -6 & & -12 & +6 & $\overline{-2}$ & +9 & -6 & +1 \\
\hline$\left(621^{2}\right)$ & +15 & -19 & -17 & +19 & -4 & -6 & +1 & -3 & +7 & -1 & +2 & - & +1 & \\
\hline$\left(531^{2}\right)$ & -4 & $\begin{array}{l}+3 \\
+\end{array}$ & $\begin{array}{r}+\quad 2 \\
\end{array}$ & -1 & $\ldots$ & & $\begin{array}{l}+\quad 2 \\
\end{array}$ & -3 & +4 & -2 & $\overline{-2}$ & $\begin{array}{l}+1 \\
+\quad 1\end{array}$ & & \\
\hline$\left(4^{2} 1^{2}\right)$ & +1 & $\begin{array}{r}+\quad 2 \\
\end{array}$ & -1 & $\ldots$ & $\ldots$ & -3 & $\begin{array}{l}+3 \\
\end{array}$ & $\begin{array}{l}+\quad 3 \\
\end{array}$ & -3 & $\ldots$ & +1 & & & \\
\hline$\left(52^{2} 1\right)$ & -13 & +2 & +9 & $\begin{array}{l}-3 \\
\end{array}$ & $\ldots$ & $\begin{array}{l}+\quad 2 \\
\end{array}$ & -1 & +3 & -3 & +1 & & & & \\
\hline$(4321)$ & +5 & $\begin{array}{l}+\quad 4 \\
+\end{array}$ & -3 & $\ldots$ & $\ldots$ & +4 & -3 & -3 & +1 & 2 & & & & \\
\hline$\left(3^{3} 1\right)$ & -1 & $\ldots$ & $\ldots$ & $\ldots$ & $\ldots$ & -2 & $\ldots$ & +1 & & Z & & & & \\
\hline$\left(42^{3}\right)$ & +4 & -2 & $\ldots$ & $\ldots$ & $\ldots$ & -2 & +1 & & & - & & & & \\
\hline$\left(3^{2} 2^{2}\right)$ & -2 & $\ldots$ & $\ldots$ & 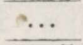 &.. & +1 & & & & & & & & \\
\hline$\left(61^{4}\right)$ & -5 & +5 & +5 & -5 & +1 & & & & & & & & & \\
\hline$\left(521^{3}\right)$ & +5 & -1 & $\begin{array}{l}-3 \\
\end{array}$ & +1 & & & & & & & & & & \\
\hline$\left(431^{3}\right)$ & -1 & -2 & +1 & & & & & & & & & & & \\
\hline$\left(42^{2} 1^{2}\right)$ & -2 & +1 & & & & & & & & & & & & \\
\hline$\left(3^{2} 21^{2}\right)$ & +1 & & & & & & & & & & & & & \\
\hline & & & & & & & & & & & & & & \\
\hline & & & & & & & & & & & & & & \\
\hline & & & & & & & & & & & & & & \\
\hline & & & & & & & & & & & & & & \\
\hline & & & & & & & & & & & & & & \\
\hline & & & & & & & & & & & & & & \\
\hline & & & & & & & & & & & & & & \\
\hline & & & & & & & & & & & & & & \\
\hline & & & & & & & & & & & & & & \\
\hline & & & & & & & & & & & & & & \\
\hline & & & & & & & & & & & & & & \\
\hline & & & & & & & & & & & & & & \\
\hline
\end{tabular}




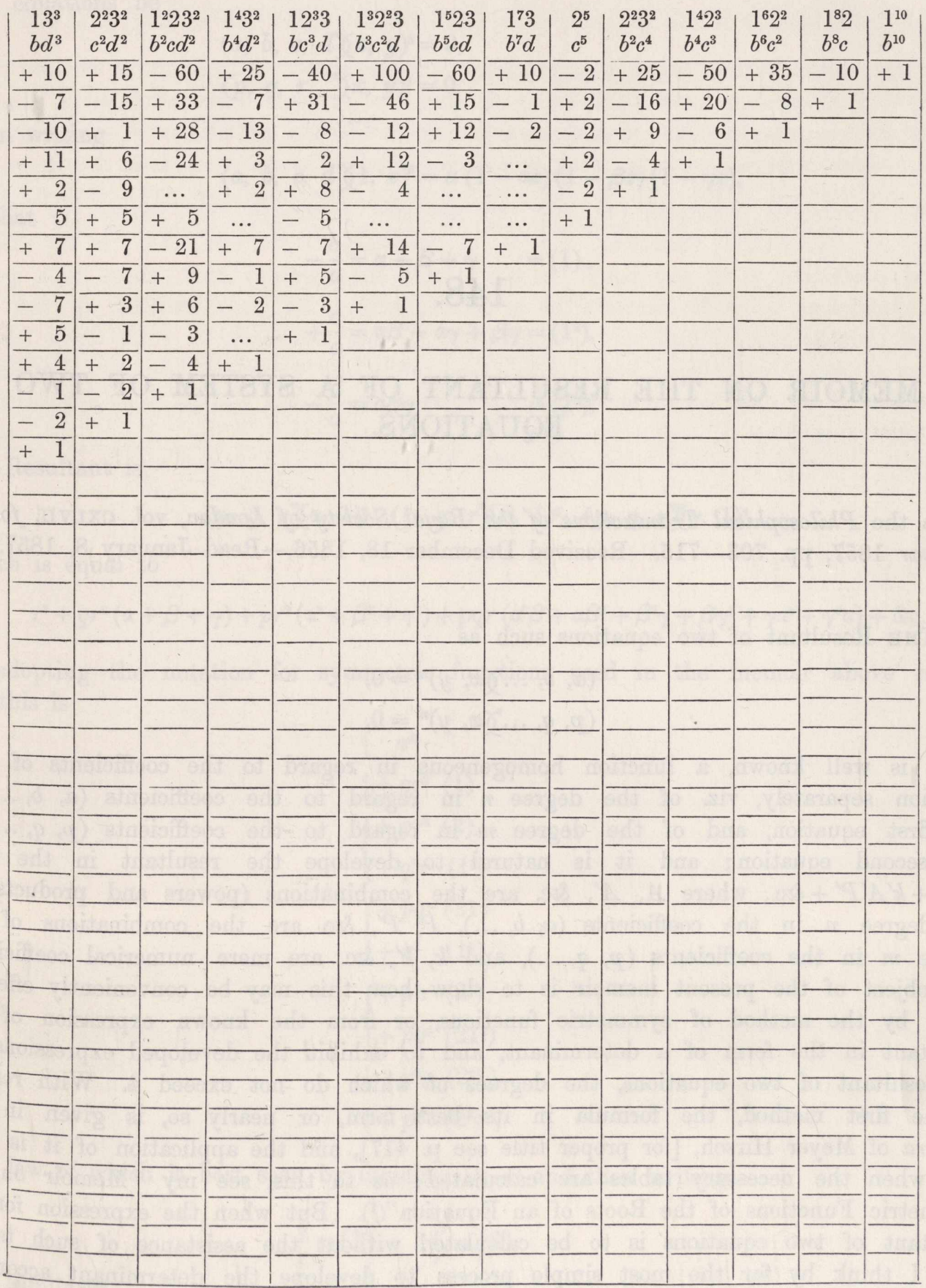

